\title{
Micromamíferos, cambio climático e impacto antrópico: ¿Cuánto han cambiado las comunidades del sur de América del Sur en los últimos 500 años?
}

\author{
Pablo Teta ${ }^{1 *}$, Anahí Formoso ${ }^{1}$, Mauro Tammone ${ }^{1}$, Daniela C. de Tommaso ${ }^{1}$, \\ Fernando J. Fernández ${ }^{2}$, Julio Torres ${ }^{1}$ y Ulyses F. J. Pardiñas ${ }^{1}$
}

\begin{abstract}
Introduction: The last 500 years of the historical era, an interval that corresponds to the concerted dispersal of European explorers, traders, and colonists around the globe, has witnessed the global disappearance of $\sim 90$ mammal species. Besides the known cases of biological extinctions, this time period was also characterized by the regional extirpations of specialized taxa and by the expansion and population growth of some opportunistic species. Several lines of evidence suggest that the current configuration of small mammal communities -i. e. richness (number of species) and diversity (distribution of species abundance)- in southern South America would have been generated in the period after the arrival of Europeans ca. 1500 AD. In this study, we reviewed the fossil record for small rodents and marsupials during the last 500 years, with emphasis on the Pampean and Patagonian regions. Based on these findings, we offer some considerations concerning the biogeography and conservation of these species.
\end{abstract}

Methods: Micromammals from archaeological and paleontological sites have provided considerable information on environmental conditions during the Quaternary in South America. In this work, we reviewed several micromammal fossil samples, mostly generated by the trophic activity of owls. The use of this kind of data involves some extrinsic and intrinsic biases that must be considered at the time to study the diversity of past communities, such as the bird involved on the accumulations, its hunting techniques, the time of the year, the size and behavior of the prey species, etc. For this work, we compared the fossil samples with more than 700 owl pellet assemblages of the same geographical areas.

Results: Richness and diversity of small mammal communities was higher prior to the deepest human impact (>0.5 ka), showing a pronounced drop in both parameters to the present. The regional extinction of some cricetid rodents and small marsupials was recorded, both in the Pampean (e. g. Bibimys torresi, Pseudoryzomys simplex) and in the Patagonian regions (e. g. Euneomys mordax, Lestodelphys halli, Tympanoctomys kirchnerorum), as well as the biological extinctions of the bat Desmodus cf. D. draculae and the cavy Galea tixiensis in the Pampas. At this same time, opportunistic species (e. g. Calomys spp., Oligoryzomys longicaudatus) showed a dramatic increase of their populations, especially in the most extensively disturbed areas.

Discussion and conclusions: In the last 500 years, significant variations occurred in the micromammal assemblages of southern South America. These changes included the dispersion and increase (in some cases extensive) of some specialized species and the regional extinctions of others, involving hundreds to thousands of kilometers of distributional extent. We suggest that the changes produced by livestock and agriculture have caused extensive habitat uniformity which was beneficial for some opportunistic taxa (e. $g$. Calomys spp., Oligoryzomys longicaudatus), facilitating their dispersion and allowing the increase of their

'Unidad de Investigación Diversidad, Sistemática y Evolución, Centro Nacional Patagónico, Puerto Madryn. Chubut, Argentina. E-mail: antheca@yahoo.com.ar(PT); formoso@cenpat.edu.ar (AF); mtammone@gmail.com (MT); danieladetommaso@yahoo.com.ar (DDT); juliomystorres@gmail.com (JT); ulyses@cenpat.edu.ar (UFJP).

${ }^{2}$ Universidad Nacional de La Plata, Facultad de Ciencias Naturales y Museo. Cátedra de Anatomía Comparada, La Plata. Buenos Aires, Argentina. E-mail: fernandezf77@yahoo.com.ar.

${ }^{*}$ Corresponding author 
populations. Anthropic disturbances also include the extensive use of fire, urbanization, and introduction of exotic forbs and grasses. Of about 46 species rodents considered as Least Concern by the IUCN and with fossil record for the last 500 years, 23 (50\%) have experienced drastic reductions in their range or abundance during the Late Holocene. At least nine taxa are completely extinct, including one marsupial, one bat and seven rodents. Similar phenomena are recorded in other areas of the Southern Hemisphere, both in continental and island contexts. This evaluation of the fossil record highlights the need to evaluate more carefully the status of some species with supposedly stable populations, but which are likely to be affected by future extensive changes in their environments (e. g. mining, expansion of the agricultural frontier).

Key words: Argentina, Didelphimorphia, extinctions, small mammals, Rodentia, Sigmodontinae.

\section{Resumen}

Diversas evidencias sugieren que la actual configuración de las comunidades -i. e. riqueza (número de especies) y diversidad (distribución de la abundancia de especies)- de micromamíferos en el sur de América del Sur se habría generado en el período posterior a la llegada de los europeos hacia 1500 AD. En este trabajo se revisa principalmente el registro fósil para pequeños roedores y marsupiales en los últimos 500 años, con énfasis en aquellos de la región Pampeana y la Patagonia. Esta evidencia se comparó con registros actuales correspondientes a más de 700 muestras de egagrópilas de estos mismos sectores geográficos. En una primera aproximación, se destaca que la riqueza y diversidad de las comunidades de micromamíferos fue mayor en la etapa previa al impacto antrópico más profundo, con una abrupta caída de ambos parámetros hacia el presente. Se sugiere que las transformaciones producidas por la ganadería y agricultura extensivas habrían provocado una uniformidad de hábitat beneficiosa para algunos taxones oportunistas, facilitando su dispersión y permitiendo el incremento, en algunos casos drástico, de sus poblaciones. En los últimos 500 años se registran variaciones significativas en la distribución de otros taxones, que en algunos casos implican extinciones locales involucrando cientos o miles de kilómetros. Sobre 46 especies de roedores consideradas como Preocupación Menor por la UICN y con registro fósil para este segmento temporal, 23 (50\%) han experimentado retracciones en sus rangos de distribución o reducciones drásticas de su abundancia. Al menos nueve taxones se habrían extinguido completamente en América del Sur, incluyendo un marsupial, un quiróptero y siete roedores. Fenómenos similares se registran en otras áreas del Hemisferio Sur, tanto en contextos continentales como insulares. Este panorama pone de manifiesto la necesidad de evaluar más cuidadosamente la situación de algunas especies con poblaciones consideradas como estables, pero susceptibles de verse afectadas frente a nuevos cambios extensivos en los ambientes (e. g. megaminería, expansión de la frontera agrícola), a juzgar por lo que indica el registro fósil.

Palabras clave: Argentina, Didelphimorphia, extinciones, pequeños mamíferos, Rodentia, Sigmodontinae.

Hacia finales del Pleistoceno y principios del Holoceno (15-8 ka [1 ka = 1000 años]) se produjo la extinción masiva de numerosas especies de vertebrados, principalmente mamíferos, muchas de las cuales superaban la media tonelada de peso (Haynes 
2009). Las causas de este fenómeno permanecen todavía poco comprendidas, aunque las principales hipótesis otorgan un papel preponderante a los cambios climáticos posteriores al Último Máximo Glacial y al impacto de la expansión del hombre (e. g. Cione et al. 2003; Borrero 2007; Haynes 2009; Barnosky y Lindsey 2010). Durante el Holoceno, las poblaciones humanas cambiaron dramáticamente los patrones regionales de biodiversidad a través de la sobreexplotación de recursos, la alteración de los hábitat originales y la introducción de especies (e. g. Steadman y Martin 2003; Turvey et al. 2011).

Una de las consecuencias inmediatas de esta situación ha sido la extinción sostenida y generalizada de plantas y animales. Para algunos autores, esta extinción representaría la continuidad del proceso iniciado a finales del Pleistoceno y cuyos efectos han sido más acusados desde el año 1500 AD (e. g. MacPhee y Flemming 1999; Turvey 2009). Según las compilaciones más recientes disponibles, durante los últimos 10 ka se habrían extinguido al menos 255 especies de mamíferos, de las cuales 90 desaparecieron en los últimos cinco siglos (MacPhee y Flemming 1999; Turvey et al. 2011). La mayoría de las especies desaparecidas eran habitantes de islas $(>70 \%)$ y, más de la mitad, roedores menores a un kilogramo de peso (MacPhee y Flemming 1999; Turvey 2009).

Hacia finales del siglo XV se produce el arribo definitivo de los europeos al Nuevo Mundo, producto de la continua exploración del Océano Atlántico con fines comerciales (Céspedes del Castillo 2009). En los dos siglos posteriores ocurre el establecimiento de las colonias, expansión y consolidación de las potencias mercantilistas europeas en Sudamérica (Founier 1998; Céspedes del Castillo 2009). Esto marcó el inicio de una serie de transformaciones económicas, tecnológicas, socioculturales y ambientales, que se profundizaron con el advenimiento de la Revolución Industrial, entre los siglos XVIII y XIX (Galeano 1971). El aumento demográfico sostenido y la necesidad creciente de materias primas que trajo aparejada esta situación, actuó como disparador de cambios sustanciales en los sistemas de cultivo, ganadería y estructura de la propiedad de la tierra, que conjuntamente con el empleo de maquinarias, permitieron un aumento en la productividad agropecuaria (Ghersa et al. 1998). El ingreso de Argentina en este mercado ocurrió hacia la segunda mitad del siglo XIX y coincidió con una importante oleada inmigratoria de colonos que ocuparon progresivamente el interior del país (Ghersa et al. 1998). A nivel global, los últimos 500 años fueron escenario de cambios climáticos significativos, incluyendo el período final de la Pequeña Edad de Hielo (PEH [siglos XIV a XIX]) y, más recientemente, del sostenido aumento de las temperaturas medias como consecuencia de la acumulación de gases de invernadero (e. g. Vimeaux et al. 2009). El impacto de estos cambios sobre la biota es bien conocido, especialmente para aves (Fraga 2003; Di Giacomo y Di Giacomo 2004; Codesido et al. 2011, 2012), mamíferos mayores (Galliari et al. 1991; Medan et al. 2011) y la vegetación (Rapoport 1996). Evidencias acumuladas en las últimas dos décadas sugieren que las comunidades de micromamíferos también cargan con el sello de estas modificaciones, acusando cambios profundos en su estructura (e. g. Pardiñas 1998, 1999a, b; Teta et al. 2005; UdrizarSauthier 2009; Fernández 2012; Pardiñas et al. 2012; Pardiñas y Teta 2013).

En este trabajo se revisaron algunas secuencias estratigráficas con fósiles de micromamíferos de yacimientos ubicados en el cono sur de América del Sur, con énfasis en aquellos de las regiones Pampeana y Patagónica de Argentina y en el segmento temporal 
de los últimos 500 años. Los depósitos estudiados presentan acumulaciones de restos de roedores cricétidos y caviomorfos y, en menor medida, marsupiales y quirópteros y fueron generados básicamente por la actividad depredadora de búhos y lechuzas. En una primera aproximación, se compararon las estructuras de las comunidades actuales (i. e. los últimos 30 años) contra aquellas correspondientes al Holoceno más tardío (i. e. $<1 \mathrm{ka}$ ) a través de dos parámetros sencillos: riqueza específica (i. e. número de especies presentes en una muestra) y diversidad específica (i. e., distribución de la abundancia de especies). Se puso especial atención en documentar retracciones distribucionales significativas (en el orden de los cientos de kilómetros)y los casos conocidos de extinciones biológicas.

En segundo lugar, se discutió el efecto que habrían tenido las modificaciones de los ambientes en el contexto de las condiciones climáticas registradas para los últimos 500 años. Finalmente, se destacan las implicancias de los hallazgos realizados en términos biogeográficos y de conservación y se contextualizan las evidencias con registros similares para otras regiones del Hemisferio Sur.

\section{Muestras estudiadas: naturaleza y limitaciones}

Las muestras de restos fósiles estudiadas en este trabajo tienen un origen mayoritario en la actividad depredadora de rapaces nocturnas; estas aves consumen cantidades significativas de pequeños mamíferos y luego expelen sus dientes, huesos y demás restos no digeridos conformando acumulaciones al pie de nidos y posaderos (Andrews 1990). Este origen implica sesgos que deben ser discutidos antes de utilizar esta fuente de evidencias como una aproximación a la riqueza y diversidad de micromamíferos en el pasado. Parte de este ejercicio consiste en asumir que tanto los hábitos de los depredadores como aquellos de las presas no han variado sustancialmente durante los últimos cientos de años, incluyendo aspectos tales como las técnicas de caza, áreas de acción o tiempos de actividad. En segundo lugar, hay que aceptar que las conclusiones obtenidas a partir de una muestra son de carácter local y que no necesariamente pueden extrapolarse regionalmente. Finalmente, debe admitirse que cada conjunto ha sido probablemente el resultado de más de un evento de depredación, involucrando desde algunos años hasta décadas. Esta última premisa permite suponer una dilución de las tendencias anuales particulares y/o catastróficas (e. g. un incremento desmedido de la abundancia de alguna especie por algún factor circunstancial), favoreciendo la emergencia de tendencias generales representativas de la comunidad depredada (e. g. Avery 1982; Andrews 1990; Stahl 1996; Pardiñas 1999a). Los alcances y limitaciones de esta metodología han sido discutidos por distintos autores (e. g. Avery 1982; Andrews 1990; Pardiñas 1999a; Fernández 2012).

Los estudios tafonómicos de algunas de las muestras fósiles consideradas en este trabajo indican que el principal agente acumulador de restos de micromamíferos en cuevas y aleros en el sur de América del Sur ha sido la lechuza de campanario, Tyto alba (e. g. Pearson y Pearson 1993; Saavedra y Simonetti 1998; Pardiñas 2000; Fernández 2012). Esta rapaz tiene un tamaño mediano (280-450 g) y habita en diversos ambientes, incluyendo zonas antropizadas y urbanas, pero con mayor frecuencia en áreas abiertas de estepa o pastizal (e. g. Taylor 1994). Usualmente, hace sus nidos en cuevas, grietas en rocas y también en construcciones humanas y, dependiendo de la disponibilidad 
de presas, su territorio de caza oscila entre 3 y $5 \mathrm{~km}^{2}$ (Taylor 1994). Tyto alba es una lechuza de hábitos crepusculares y nocturnos, que captura a sus presas principalmente en ambientes abiertos; sus hábitos dietarios son oportunistas, alimentándose principalmente de micromamíferos y otros pequeños vertebrados, cuyo tamaño promedio varía entre 10 y 360 g (Taylor 1994 ; Bellocq 2000). Las acumulaciones de elementos óseos y dentarios que genera esta lechuza exhiben una baja modificación por corrosión digestiva y fractura y una elevada representación de casi todas las piezas anatómicas (Andrews 1990). De estas características pueden deducirse varios sesgos, que van desde la escala local de sus conjuntos (por su radio de acción limitado) y la sub-representación de roedores diurnos y/o grandes en tamaño corporal (> 250 g) hasta la sobre-representación de especies pequeñas, solitarias y nocturnas y que ocupan ambientes abiertos y con elevada exposición a la depredación (Pardiñas 2000). En América del Sur, sus principales presas son los roedores sigmodontinos, algunos roedores caviomorfos y marsupiales pequeños (Bellocq 2000; Pardiñas y Cirignoli 2002; Bó et al. 2007 y la literatura allí citada).

Al menos parcialmente, muchos de los sesgos indicados en el párrafo precedente, pueden ser controlados si se comparan muestras fósiles y actuales producidas por las mismas aves. Así, partiendo de la premisa de una invariancia de las conductas de depredadores y presas durante el Holoceno (i. e. ni las lechuzas han cambiado sus principales hábitos tróficos, ni los pequeños mamíferos han cambiado sus principales pautas de conducta durante los últimos miles de años), los contrastes entre las acumulaciones pretéritas y recientes pueden interpretarse confiablemente como reflejo de variaciones ambientales.

\section{Comunidades de micromamíferos durante el Holoceno más tardío: casos de estudio}

De las dos regiones focales de esta contribución se han seleccionado algunos yacimientos con restos de micromamíferos que, con su correspondiente contraparte actual, permiten visualizar la evolución de la riqueza y diversidad específicas durante los últimos 500 años (Figs. 1-4). Esta selección de localidades no es caprichosa sino operativa, ya que se trata de aquellas mejor conocidas y más robustas por control cronológico (en número y calidad de dataciones radiocarbónicas) y tamaño de muestra (a la hora de evitar los sesgos derivados del análisis de muestras pequeñas).

Región Pampeana, sudeste de Buenos Aires. La evidencia disponible para esta región incluye dos tipos de registro: yacimientos arqueológicos (Cueva Tixi y Divisadero Monte 6) y paleontológicos (Centinela del Mar; Fig. 1). En ambos casos, los estudios tafonómicos sugieren que los restos de micromamíferos fueron ingresados básicamente por la acción trófica de aves rapaces, aunque en Divisadero Monte 6 algunos roedores $>100 \mathrm{~g}$ (e. g. Ctenomys, Caviidae) podrían haber sido incorporados para consumo humano (Silveira et al. 2010).

El ensamble aquí considerado para Cueva Tixi corresponde a los restos de micromamíferos recuperados en el "Estrato B" según los datos de NISP brindados por Quintana (2001a: tabla 1). Este autor indica la presencia de dos marsupiales, ocho cricétidos y tres caviomorfos para este nivel estratigráfico, con una cronología de $170 \pm$ 60 ar AP (años radiocarbónicos antes del presente). Para Divisadero Monte 6 el detalle del material estudiado corresponde a Teta et al. (2013: tabla 1), quienes indican un 
ensamble conformado por 11 cricétidos con una edad de $540 \pm 60$ ar AP. A estos deben agregarse un marsupial y dos caviomorfos de acuerdo a Silveira et al. (2010).

Para la región de Centinela del Mar consideramos dos depósitos, cuyos detalles más significativos se anotan en Pardiñas (1999a; véase también Pardiñas y Tonni 2000).

El depósito cronológicamente más antiguo corresponde al relleno de una oquedad formada a expensas de sedimentitas del tope de la secuencia estratigráfica; una datación efectuada mediante acelerador de partículas sobre hemimandíbulas seleccionadas de Reithrodon auritus brindó un fechado de $565 \pm 50$ años ar AP (AA30465), con edades calibradas ( \pm 1 sigma) en años calendario entre 526-632. Otra muestra corresponde a una plataforma de erosión con relleno estratificado, que fue excavada en tres niveles artificiales de tres $\mathrm{cm}$ de espesor cada uno. Un fechado por acelerador de partículas sobre hemimandíbulas de $R$. auritus brindó $250 \pm 65$ años ar AP (AA30466), con un rango 0-314 en años calendarios ( \pm 1 sigma; Pardiñas 1999a). Ambos ensambles son ricos en roedores cricétidos y, en menor medida, caviomorfos y marsupiales.
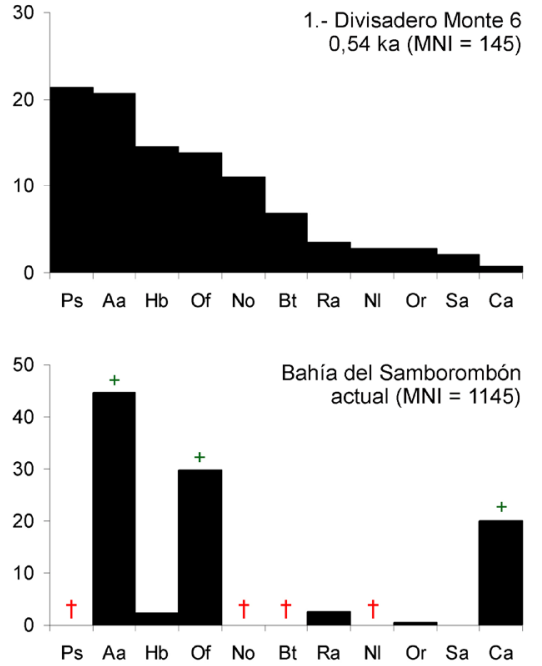
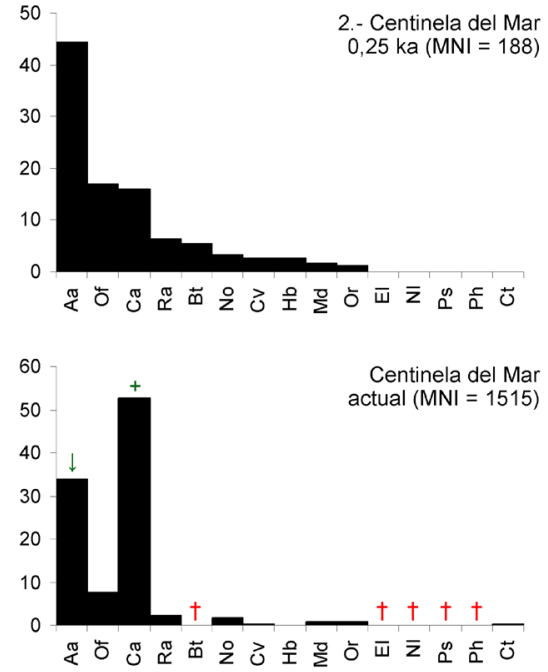
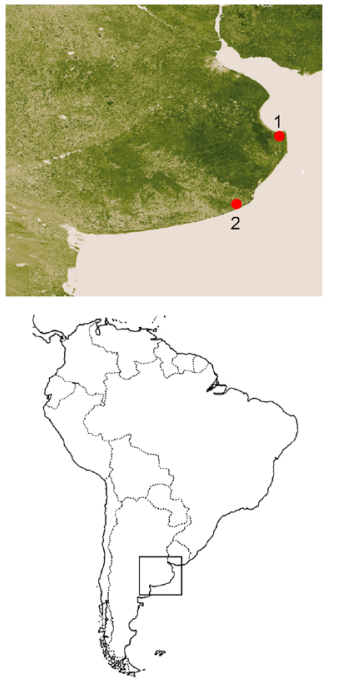

Figura 1.Muestras fósiles (arriba) y actuales (abajo) seleccionadas para la Región Pampeana, sudeste de Buenos Aires, indicando cambios en la abundancia de micromamíferos (expresada como porcentaje del número mínimo de individuos $[\mathrm{MNI}])$. Las cruces señalan extinciones; la flecha hacia abajo declinaciones en la abundancia y el signo + un aumento en la representación. Para una referencia de las abreviaturas, véase el Apéndice 1.

En los ensambles estudiados hay que distinguir tres situaciones ambientales: 1) Aquellos emplazados en el ambiente serrano del sistema de Tandilia (Cueva Tixi). 2) Aquellos en el ambiente costero del sudeste (Centinela del Mar), y 3) Aquellos emplazados en el ambiente costero cercano a la Bahía de Samborombón (Divisadero Monte 6). Esta diferenciación no es menor, ya que pueden existir características particulares dependientes de estas situaciones ambientales (cf. Pardiñas et al. 2010a).

Globalmente, cualquiera de los ensambles del Holoceno tardío del sudeste bonaerense se caracteriza por ser más rico en número de especies que sus contrapartes actuales. Se registran varios casos de cricétidos extintos regionalmente: Bibimys torresi, Pseudoryzomys simplex, Phyllotis sp., Eligmodontia sp. y Necromys lasiurus (Pardiñas 1995, 1999a, b). A estos se suman otros taxones que podrían estar representando extinciones biológicas (e. g. el quiróptero Desmodus cf. D. draculae y el caviomorfo Galea tixiensis; Pardiñas y Tonni 2000; Quintana 2001a). Por otra parte, al menos Calomys spp. muestra un incremento dramático -también verificado en otras regiones de la Pampasia (e. g. Pardiñas 1999a; Pardiñas et al. 2010a, b; González-Fischer et al. 
2012; Fig. 1)- mientras que parece ocurrir algo similar, aunque a menor escala, con Oligoryzomys flavescens en el ámbito serrano (Pardiñas 2000; Pardiñas et al. 2010a).

Patagonia noroccidental. El registro fósil para esta región corresponde casi por completo a contextos arqueológicos. Por lo menos dos de éstos, las cuevas Traful I (CTI) y Epullán Grande (LL), contienen secuencias razonablemente continuas para los últimos 10 ka (Pardiñas y Teta 2013). Estos depósitos, además, están emplazados en el Distrito Subandino $(\mathrm{CTI})$ y el Distrito Occidental en un área de interdigitación con el Distrito Oriental y el Ecotono Rionegrino del Monte austral (LL; para la definición de las unidades fitogeográficas véase León et al. 1998; Fig. 2), y por lo tanto, podrían ser ambientalmente representativos de dos unidades florísticas mayores (Fig. 2). En otros yacimientos (e. g. Cueva y Paredón Loncomán, Cuevas Sarita I, II y IV, Casa de Piedra de Ortega) las evidencias disponibles están limitadas al Holoceno tardío (e. g. Pardiñas 1999a; Pardiñas et al. 2005; Teta et al. 2005). En su mayoría se trata de acumulaciones generadas por aves rapaces, aunque con un importante componente antrópico para los roedores de mayor porte, especialmente Caviidae y Ctenomyidae (Pardiñas 1999a; Teta et al. 2005).

Las secuencias de CTI y LL muestran que el conjunto de especies prácticamente no cambió, tanto en términos de riqueza como diversidad, durante los últimos $10 \mathrm{ka}$, contrastando en forma drástica con la estructura actual de los ensambles (véase abajo).

Dos hipótesis alternativas, aunque no necesariamente excluyentes, podrían explicar esta situación de estabilidad comunitaria durante la mayor parte del Holoceno. Por un lado, podría suponerse que los cambios climáticos ocurridos no tuvieron la magnitud suficiente para provocar reorganizaciones significativas en las comunidades. Por el otro, que las especies de pequeños mamíferos fueron resilientes, pese a la ocurrencia de cambios ambientales, independientemente de la intensidad de los mismos (Pardiñas 1999a; Pardiñas y Teta 2013). En contraste con lo observado para el Holoceno, las muestras actuales indican una reestructuración comunitaria profunda, que se traduce en una acusada disminución de la diversidad, dada por la dominancia de unos pocos taxones oportunistas y una pérdida general de sigmodontinos de tamaño mediano a grande (Teta et al. 2005; Pardiñas y Teta 2013). En efecto, la riqueza varió de $S=14$ (CTI) o 13 (LL) taxones hacia 0,5 ka a $\mathrm{S}=8$ y 12 para muestras actuales de esas mismas regiones, respectivamente (Pardiñas y Teta 2013; Fig. 2). Los ensambles recientes están signados por frecuencias elevadas de Abrothrix longipilis, A. olivacea y Oligoryzomys longicaudatus en los ambientes arbustivos y ecotonales del oeste y por Eligmodontia spp. hacia las estepas semiarbustivas orientales (Teta et al. 2005; Pardiñas y Teta 2013).

En algunos sectores, la suma de estos taxones supera el $70 \%$ del total de la comunidad (Fig. 2). Al menos el marsupial Lestodelphys halli y el sigmodontino Euneomys mordax han desaparecido local o regionalmente, contra sus registros durante buena parte del Holoceno. Otras especies acusan disminuciones moderadas a drásticas en su representación (e. g. Ctenomys sociabilis, Euneomys chinchilloides, Reithrodon auritus; Fig. 2).

Patagonia central, valle del río Chubut. Para este sector se cuenta con registros para dos sitios paleontológicos, ambos ubicados en el área de influencia del río Chubut: Lle Cul 
y Alero Las Plumas (Pardiñas et al. 2000, Udrizar Sauthier 2009; Pardiñas et al. 2012; Fig. 3). Ambos sitios fueron generados por la actividad depredadora de aves rapaces, mayoritariamente T. alba.

Lle Cul se encuentra en el valle inferior del río Chubut; los restos estudiados formaban parte del relleno de una grieta, sellada cuspidalmente por yeso. Una datación radiocarbónica sobre el material postcraneano brindó una edad de $1830 \pm 70$ ar AP. El ensamble de micromamíferos estaba integrado por seis roedores sigmodontinos, dos caviomorfos, dos quirópteros y dos marsupiales, destacándose por sus abundancias Calomys musculinus (35,2\%) y Eligmodontia sp. (33,4\%). Pardiñas et al. (2000) incluyen una comparación con una muestra actual de egagrópilas de $T$. alba procedente de la misma localidad y representativa de un año de muestreo (García Esponda et al. 1998).

Entre la muestra fósil y la actual se observa una drástica caída de la diversidad específica, con una dominancia cercana al 95\% para Calomys musculinus y la extinción regional del marsupial Lestodelphys halli (Fig. 3).
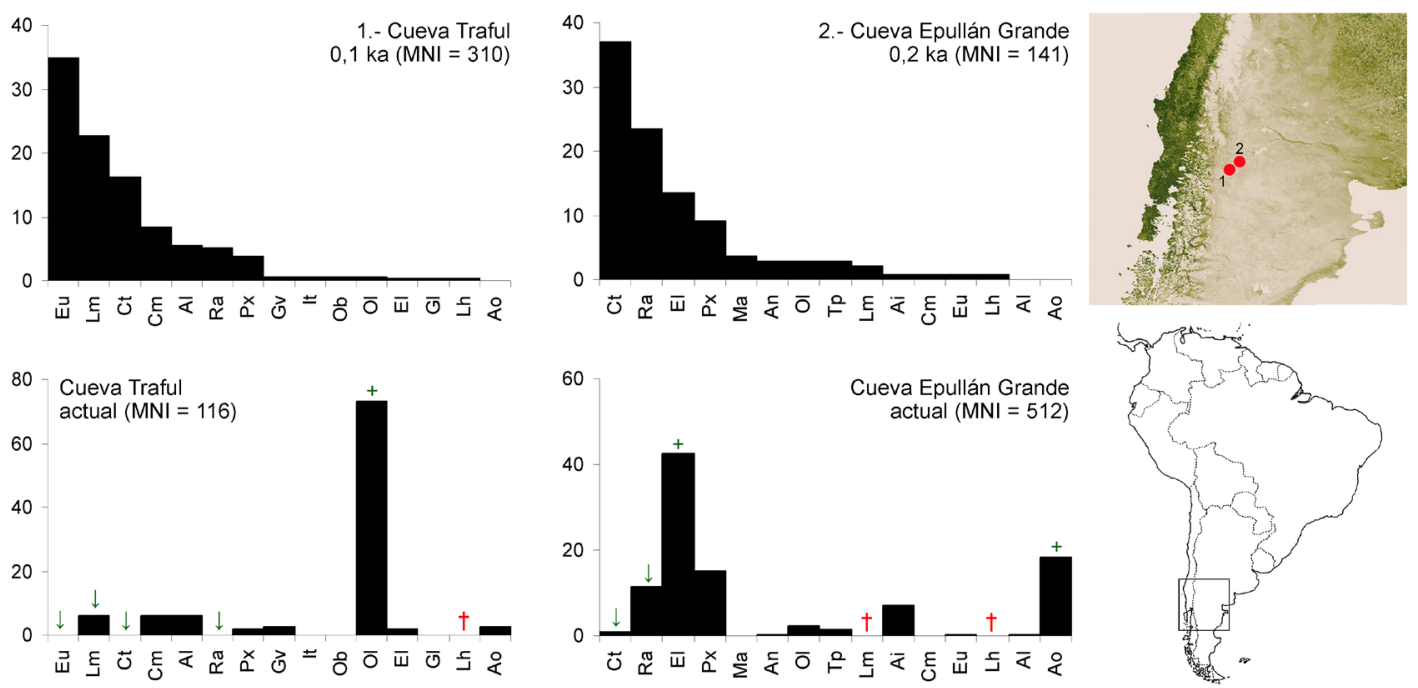

Figura 2. Muestras fósiles (arriba) y actuales (abajo) seleccionadas para la Patagonia noroccidental, indicando cambios en la abundancia de micromamíferos (expresada como porcentaje del número
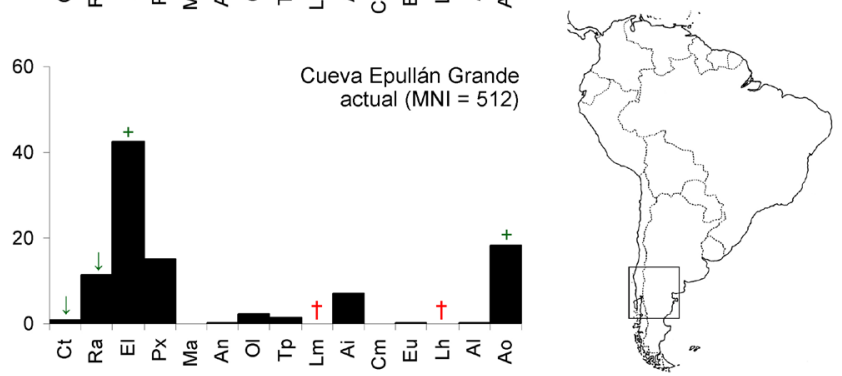
mínimo de individuos $[\mathrm{MNI}])$ Las cruces señalan extinciones; la flecha hacia abajo declinaciones en la abundancia y el signo + un aumento en la representación.Para una referencia de las abreviaturas, véase el Apéndice 1.

Alero Las Plumas se ubica hacia el valle medio del río Chubut, habiendo sido recuperados los restos a partir de un depósito unicomponente de $20 \mathrm{~cm}$ de espesor datado en $410 \pm$ 80 ar AP. El ensamble estuvo integrado por 13 especies, con dominancia de Eligmodontia sp. (56,6\%) y Ctenomys sp. (17,9\%). Las muestras actuales revelaron valores de riqueza específica más bajos que en la muestra fósil: al menos cuatro sigmodontinos, Abrothrix longipilis, Loxodontomys micropus, Notiomys edwardsii y Oligoryzomys longicaudatus y 1 caviomorfo, Tympanoctomys kirchnerorum, se extinguieron localmente (Pardiñas et al. 2012; Fig. 3). En líneas generales, la retracción de estos taxones parece tener una componente oeste-noroeste, hacia contextos ambientales actualmente más fríos y húmedos.

Patagonia sudoccidental. Dos yacimientos ubicados en el noroeste de la provincia de Santa Cruz cuentan con secuencias arqueológicas que se extienden hasta el Holoceno medio. Alero Destacamento Guardaparque (ADG) es un sitio bajo roca que se emplaza dentro del Parque Nacional Perito Moreno, hacia un área de estepa. Su secuencia 
estratigráfica incluye siete capas, de las cuales la 4(1a) ha sido fechada en $890 \pm 70$ ar AP [LP-288], estimándose el tope en 0,3 ka. Cerro Casa de Piedra 5 (CCP5) se ubica algo al oeste de ADG, en una matriz que incluye elementos boscosos y ecotonales (Fig. 4). La cronología para este sitio, donde también se reconocieron siete capas, se extiende entre $6780 \pm 110$ años ar AP (capa 7) y $2550 \pm 90$ años ${ }^{14} \mathrm{C}$ AP (capa 1b). Para este trabajo, se consideraron las capas que suprayacen a la 1b, referibles al Holoceno más tardío (Pardiñas 1998, 1999a).

Las muestras más recientes para ambos yacimientos indican una riqueza de 10 a 11 especies, incluyendo 9-10 sigmodontinos y un caviomorfo. Si bien este número no cambia para los conjuntos actuales, existen algunas diferencias significativas cuando se consideran las frecuencias para cada especie (Pardiñas 1998, 1999a). El ensamble del Holoceno tardío en ADG está dominado por Ctenomys cf. C. magellanicus (36,5\%) y Reithrodon auritus (14,9\%). En fuerte contraste, las muestras de egagrópilas actuales para este sitio señalan una dominancia de Abrothrix olivacea, con una participación superior al 50\%. Además, desaparece localmente Loxodontomys micropus y se verifica una fuerte caída en la frecuencia de $R$. auritus (Pardiñas 1999a). A diferencia de lo indicado para la Patagonia noroccidental, los valores para Euneomys chinchilloides no muestran alteraciones a lo largo del Holoceno, incluyendo el momento actual (Fig. 4). El conjunto para CCP5, si bien es menos aplicable al segmento discutido en esta contribución en términos cronológicos, muestra una situación comparable a ADG, con un incremento reciente y dramático de A. olivacea y una caída en L. micropus. Para este último sigmodontino, su frecuencia en las muestras actuales es casi 4 veces menor que en las muestras arqueológicas (Pardiñas 1998, 1999a; Fig. 4).

En CCP5, hacia los 2,5 ka se registra el sigmodontino Abrothrix lanosa. Si bien esta especie no se detecta en los ensambles del Holoceno más tardío, existe al menos un registro obtenido por trampeo para finales del siglo XIX en el valle del río Tucu Tucu, $\sim 70$ km S del Parque Nacional Perito Moreno (Allen 1905). Muestreos recientes realizados en esta región y el análisis de numerosas muestras de egagrópilas del noroeste de Santa Cruz (Formoso 2013) han fallado en detectar a esta especie, que podría considerarse como localmente extinta.

\section{Patagonia Austral}

Los restos de roedores estudiados para este sector provienen de tres yacimientos, dos arqueológicos (Orejas de Burro 1, Cóndor 1) y uno paleontológico (Puesto Pali Aike), emplazados hacia el extremo sudoriental de la provincia de Santa Cruz. Como parámetro actual, se utilizaron cuatro muestras de egagrópilas que cubren esta misma región (Pardiñas et al. 2011).

En las comunidades fósiles se registran 11 especies de roedores, pero sólo ocho en las actuales. Tres especies, exclusivas de los ensambles holocénicos, hacen esta diferencia, incluyendo a Abrothrix lanosa, Chelemys macronyx y Loxodontomys micropus. En tanto se trata de taxones vinculados con ambientes forestados y ecotonales, parece más probable que su presencia extra-limital se vincule con un pulso frío y húmedo, tal como ha sido discutido por Pardiñas et al. (2011). En la actualidad, el ambiente está dominado por una estepa graminosa semiárida, que acusa los efectos de más de 100 años de altas cargas ovinas. Llamativamente, tanto las muestras fósiles como actuales 
están dominadas por las mismas especies, Abrothrix olivacea, Euneomys chinchilloides y Reithrodon auritus (Pardiñas et al. 2011). Es decir, no parece haber habido cambios sustanciales en las relaciones de dominancia, más allá del evidente impacto antrópico, una situación que contrasta con la evolución de las comunidades en otras áreas de Patagonia (véase arriba).

Declinación y extinción en tiempo reciente. A juzgar por el registro fósil, la extinción regional de pequeños mamíferos involucró retracciones sobre áreas más extensas en la región Pampeana que en la Patagonia (e. g. Pardiñas 1999a, b; Pardiñas et al. 2010b; Pardiñas et al. 2012; Fig. 5). En esta última, por el contrario, los cambios se tradujeron más bien en una disminución de la diversidad antes que de la riqueza (véase arriba). Sin embargo, en algunos sectores geográficos se registraron extinciones regionales, como es el caso del curso medio del río Chubut, en la Patagonia central (Pardiñas et al., 2012) o el sudoeste del Neuquén (Pardiñas y Teta 2013; Fig. 6). Para este último sector, la extinción local de Ctenomys sociabilis se ha vinculado, además, con una disminución de su diversidad génica (Chan et al. 2005, Chan y Hadly 2011).

Tanto en Pampa como en Patagonia, con unas pocas excepciones, la mayoría de las extinciones de micromamíferos ocurrieron sobre poblaciones periféricas, un fenómeno también advertido para los octodóntidos en Chile central (Saavedra y Simonetti 2003). Si bien esta situación es esperable, dado que los márgenes de la distribución de una especie se asocian con tamaños de población reducidos y hábitats sub-óptimos (Brown 1984), existen estudios con otros mamíferos que contradicen esta idea. Lomolino y Channell (1995), con ejemplos de distintas partes del mundo, indicaron que el colapso en el área de distribución de 23 de 31 especies de mamíferos de tamaño pequeño a grande ocurrió desde el centro hacia la periferia. Si se aplica este escenario al caso pampeano, esta bien podría ser la situación de las especies de Necromys ( $N$. lasiurus [Fig. 5] y N. obscurus) o de Monodelphis dimidiata, cuyas poblaciones actuales persisten en áreas que resultan marginales a sus distribuciones históricas.

Para otras especies, si bien sus rangos de distribución han variado escasa o nulamente, no puede decirse lo mismo de su abundancia. Un caso que ejemplifica esta aseveración es el de Euneomys chinchilloides en el noroeste de la Patagonia. Durante más de 20 ka -esto es, desde el pico del Último Máximo Glacial- esta rata fue dominante en las comunidades (Pearson 1987; Pearson y Pearson 1993; Pardiñas y Teta 2013; Tammone et al. enviado), para casi desaparecer durante el Holoceno más tardío. Las causas de este fenómeno, que preliminarmente podrían vincularse con el impacto antrópico en la región, han sido discutidas por varios autores (e. g. Pearson 1987; Monjeau 1989; Rebane 2002; Pardiñas y Teta 2013), aunque sus detalles distan de ser claros. Situaciones menos dramáticas, pero igualmente notorias, son la disminución en la abundancia de Reithrodon auritus en algunos sectores de Pampa y Patagonia y de Necromys spp. en Pampa (e. g. Pardiñas 1999a; Galliari y Pardiñas 2000; Teta et al. 2005; Pardiñas y Teta 2013).

Turvey (2009) listó cinco especies de mamíferos que se habrían extinguido durante el Holoceno más tardío en América del Sur continental, incluyendo un marsupial (Cryptonanus ignitus [extinto hacia 1962]), un quiróptero (Desmodus cf. D. draculae 
[pre-1820]) y tres roedores (Cuscomys oblativus [pre-1532], Lagostomus crassus [pre1910] y Juscelinomys candango [ 1960]). Sin embargo, este autor omitió otros cuatro taxones que no tienen registros desde hace al menos 50 años, que son los roedores Galea tixiensis ( 1862; cf. Quintana 2001b), Gyldenstolpia fronto chacoensis ( 1896; cf. Pardiñas et al. 2008), Necromys punctulatus ( 1913; cf. Voss 1991) y Octodon pacificus ( 1959; cf. Hutterer 1993). Esta situación pone a América del Sur a la cabeza de las extinciones continentales durante el Holoceno más tardío (Fig. 6), quedando apenas por debajo de contextos insulares como el de Australia, con casi 20 especies desaparecidas desde 1500 AD (Turvey 2009). Este no es un dato menor, si se tiene en cuenta que en compilaciones previas no se indicaban pérdidas de mamíferos para los últimos 500 años en este mismo sector (cf. MacPhee y Flemming 1999). Si a esto se suman los más de 40 taxones extinguidos en islas (e. g. Galápagos, Indias Occidentales, Malvinas; MacPhee et al. 1999; Turvey 2009), la situación para el Neotrópico resulta por mucho más crítica que en otras partes del globo.

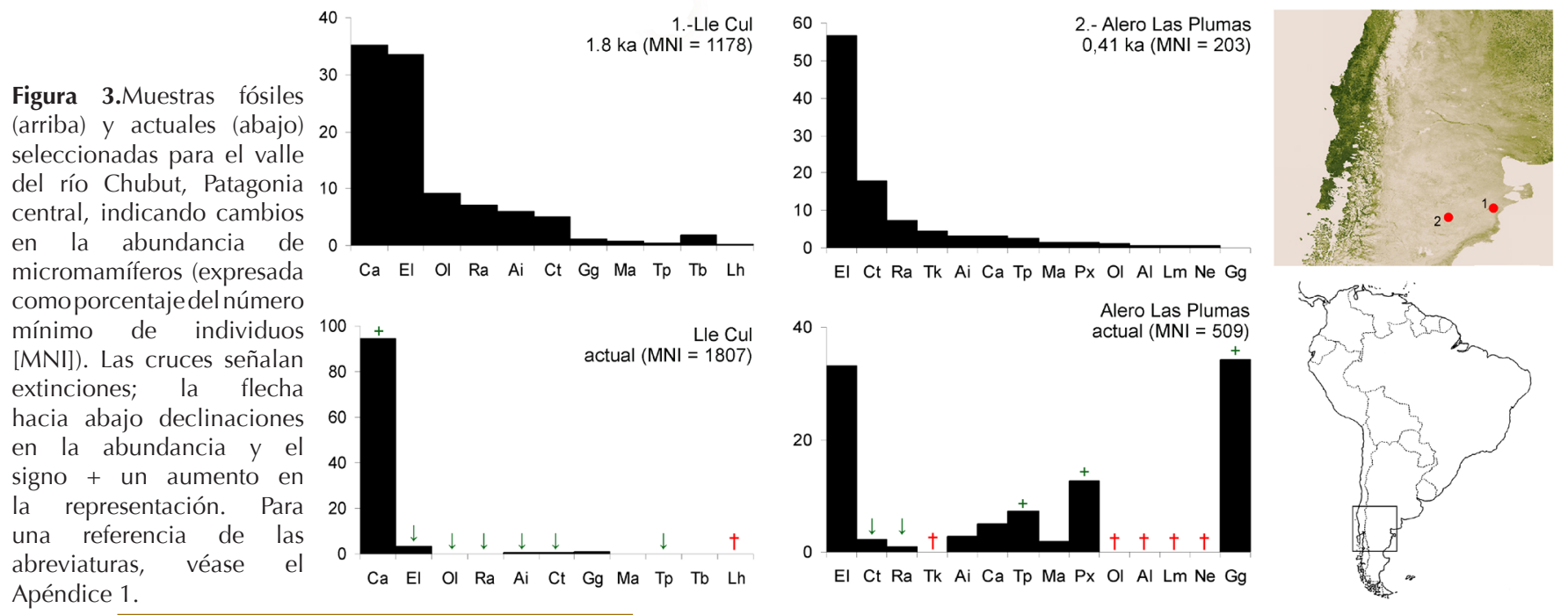

El auge de los oportunistas. Uno de los sellos distintivos de las comunidades actuales de micromamíferos en las regiones Pampeana y Patagónica es que están dominadas por una o dos especies de roedores sigmodontinos (e. g. Pardiñas 1999a; Teta et al. 2010, González-Fischer et al. 2012). Hay un número definido de síntomas que caracterizan a las situaciones de disturbio en los ecosistemas -y que se reflejan en la composición de los ensambles- de los cuales los mejor documentados son la reducción en la riqueza, reducción en el tamaño medio de los individuos y dominancia de especies oportunistas (e. g. Gray 1989). Por lo general, la riqueza se reduce a expensas de la desaparición de aquellos taxones más especializados y que no son capaces de adaptarse rápidamente a los ambientes cambiantes (e. g. McKinney y Lockwood 1999, Devictor et al. 2008). Bajo estas circunstancias, por el contrario, las especies oportunistas suelen verse favorecidas, ya que encuentran condiciones propicias para aumentar sus poblaciones y expandirse. Por lo general, las formas oportunistas tienen un tamaño pequeño y un elevado potencial de reproducción, que les permiten transformarse rápidamente en elementos dominantes (Gray 1989; McKinney y Lockwood 1999; Devictor et al. 2008). 
La situación del género Calomys durante el Holoceno en Pampa y Patagonia constituye un ejemplo paradigmático de lo previamente reseñado (Figs. 1 y 2). Estos roedores incluyendo las dos especies más australes, C. laucha y C. musculinus- tienen un tamaño pequeño $(<25 \mathrm{~g})$ y un período reproductivo extendido, al menos en la Región Pampeana (Mills et al. 1992). Distintos autores han vinculado el aumento en las poblaciones de Calomys con la conversión de los pastizales en agroecosistemas y la consecuente homogenización de los ambientes (e. g. Pardiñas 1999a; Pardiñas et al. 2010a, b; Bilenca et al. 2012; González-Fischer et al. 2012). Para ambas especies la expansión reciente de su distribución en la Región Pampeana se ve reflejada en la estructura genética de sus poblaciones (Chiappero et al. 2002a, b) y en información ecológica (Bilenca y Kravetz 1995). A una escala menor, C. musculinus se ha convertido en la especie dominante en algunos valles fluviales de varios ríos patagónicos, donde el paisaje original fue mayormente sustituido por pasturas para el ganado y campos de cultivo (Pardiñas et al. 2000; de Tommaso et al. en prensa).

En la Región Patagónica, la generalización de prácticas ganaderas extensivas y el consecuente sobrepastoreo se han traducido en una progresiva arbustización y en una disminución de la cobertura herbácea y aumento del suelo desnudo (León y Aguiar 1985; Ares et al. 1995; Perelman et al. 1997; Bertiller y Bisigato 1998). Esta transformación del paisaje natural durante la última centuria parece haber disparado incrementos en la frecuencia de ciertos taxones. Tales son los casos de Abrothrix olivacea en los ambientes ecotonales occidentales y de Eligmodontia spp. en las estepas arbustivas orientales (e. g. Monjeau 1989; Pardiñas 1999a; Teta et al. 2005). La dominancia en los conjuntos actuales de $A$. olivacea también ha sido verificada en las estepas arbustivas y herbáceas del sudoeste de Mendoza, donde este roedor aumentó considerablemente su participación en los ensambles con respecto a su devenir holocénico (Fernández 2012). Del mismo modo, Eligmodontia se ha convertido en el taxón más común en muestras actuales del noroeste argentino, en áreas modificadas por la ganadería ovina y caprina (Ortiz 2001; Ortiz et al. 2011). Un fenómeno similar ocurre con Oligoryzomys longicaudatus, que al menos localmente parece haber incrementado drásticamente su abundancia en sectores dominados por la exótica rosa mosqueta (Rosa rubiginosa), un arbusto en el que encuentra refugio y alimento (Pardiñas y Teta 2013). Al igual que en el caso de Calomys spp., los sigmodontinos aquí discutidos son también de tamaño pequeño y elevado potencial reproductivo (e. g. Pearson et al. 1987; Guthmann et al. 1997).

¿Impacto antrópico o cambio climático? Distintos autores han vinculado los procesos de extinción y declinación de micromamíferos en el sur de América del Sur en tiempos históricos con el impacto de actividades de origen antrópico. Entre estas, las más relevantes -por su extensión y potencial capacidad modificadora- fueron la introducción de ganado, la quema de pastizales, la extracción de leña, la diseminación de malezas, la conversión de los pastizales en agroecosistemas y la urbanización creciente (e. g. Soriano et al. 1992; Ghersa et al. 1998; Morello et al. 2000). Las poblaciones de vacas (Bos taurus) y caballos (Equus caballus) cimarrones se volvieron muy abundantes en los campos de la Región Pampeana poco después de sus introducciones hacia mediados del siglo XVI (Giberti 1986), ejerciendo efectos negativos sobre el suelo y la vegetación 
(Rapoport 1996; Ghersa et al. 1998). Sin embargo, los cambios estructurales más significativos ocurrieron hacia finales del Siglo XIX y primeros años del Siglo XX, con la conversión extensiva de los pastizales naturales a cultivos de cereales y oleaginosas (Ghersa et al. 1998). El impacto de estos cambios sobre la fauna ha sido repetidamente destacado, incluyendo la virtual extinción de varios ungulados nativos y carnívoros tope (Galliari et al. 1991). Teta et al. (2013) plantearon que el empleo de fuego, cuya frecuencia se intensificó hacia la etapa ecuestre, habría sido un elemento determinante en la reorganización de los ensambles, al menos en la Región Pampeana. La quema de pastizales en este sector fue una constante durante los siglos XVII y XVIII, tal como lo indican los relatos de viajeros y naturalistas (e. g. F. de Azara, C. Darwin). Aunque poco sabemos experimentalmente sobre el potencial impacto de los incendios sobre las comunidadesde micromamíferos pampeanos, los datos disponibles indican un efecto negativo, especialmente sobre la riqueza y la densidad de individuos (Comparatore et al. 1996). En ambientes sucesionales post-fuego en arbustales de Entre Ríos, Calomys parece ser uno de los primeros colonizadores (Marconi 1988). Luego de incendios en jarillales del monte en el nordeste del Chubut, Eligmodontia ha mostrado picos de frecuencia (Osorio 2003) y una situación similar se ha planteado para el Monte de Mendoza (Ojeda 1989).

Figura 4. Muestras fósiles (arriba) y actuales (abajo) seleccionadas para la Patagonia sudoccidental, indicando cambios en la abundancia de micromamíferos (expresada como porcentaje del número mínimo de individuos [MNI]). Las cruces señalan extinciones; la flecha hacia abajo declinaciones en la abundancia y el signo + un aumento en la representación. Para una referencia de las abreviaturas, véase el Apéndice 1.
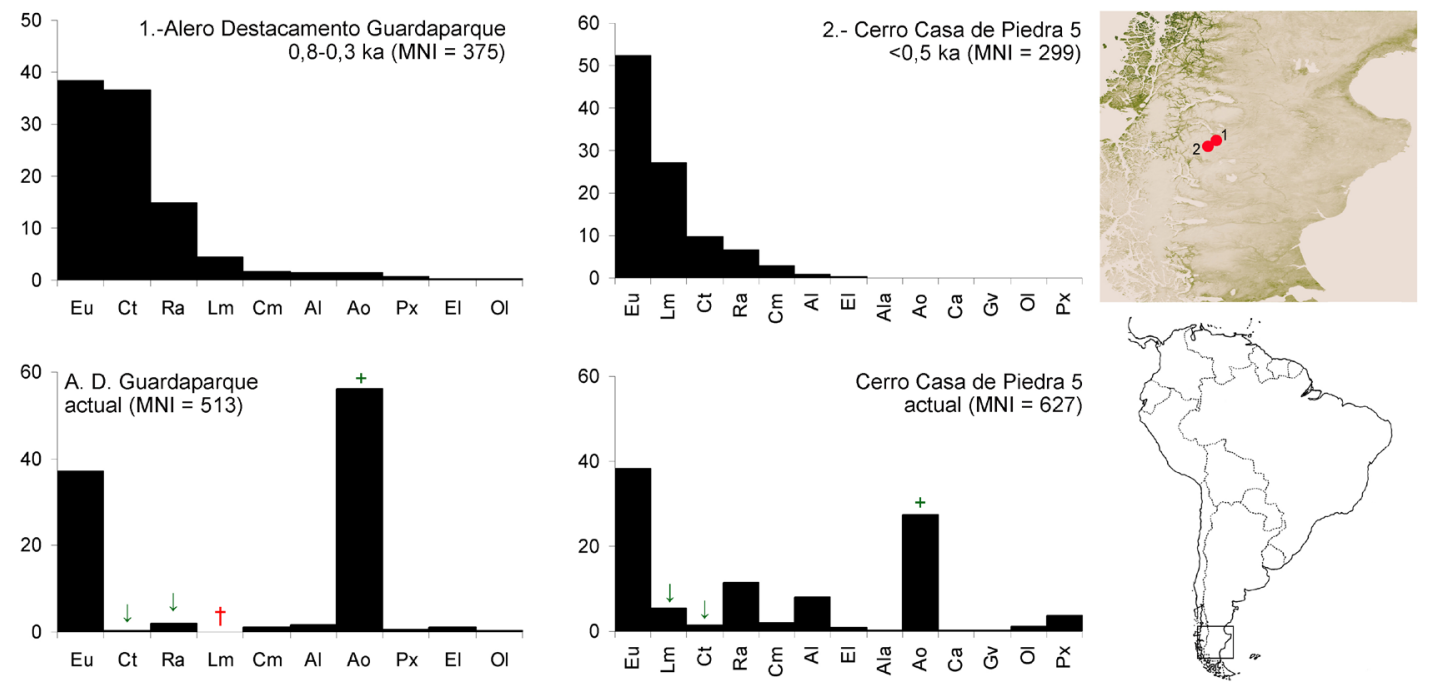

Para la Región Patagónica, el mayor impacto se produjo a partir de las grandes concentraciones de ganado ovino que tuvieron su acmé en la década de 1950 (Aagesen 2000) y, más localmente, en el desarrollo de oasis agrícolas en los valles fluviales (e. g. Williams 1975). El efecto de las ovejas no sólo se reflejó en el sobrepastoreo y consecuente desertización, sino también en la compactación de los suelos, que podría haber afectado la viabilidad de las poblaciones de algunos roedores subterráneos (e. g. Ctenomys spp., cf. Osgood 1943; Tympanoctomys kirchnerorum, cf. UdrizarSauthier et al. 2009, Pardiñas et al. 2012).

El crecimiento de las áreas urbanas, especialmente durante el último siglo, añade un factor antrópico más de modificación de los ecosistemas naturales. Este proceso, que suele ocurrir lentamente, se caracteriza por su irreversibilidad y por producir una 
considerable reducción de los servicios ecológicos (Morello et al. 2000). El efecto de la urbanización sobre las comunidades de micromamíferos ha sido poco estudiado en América del Sur, pero la evidencia disponible indica que produciría un reemplazo prácticamente total de las especies nativas por roedores comensales exóticos, como Rattus spp. y Mus musculus, al menos en aquellos sectores más profundamente modificados (Cavia et al. 2008; Teta et al. 2013). En el estudio de los ensambles de roedores en un gradiente urbano-rural, Hercolini (2007) indicó que los mayores valores de diversidad se registraban hacia contextos intermedios, en áreas suburbanas (véase también Teta et al. 2013). Esta situación estaba dada por la dominancia de uno o dos taxones en los extremos y una participación más equitativa de las mismas especies en áreas de transición (Hercolini 2007).

Más en general, en la mayoría de los casos no hay evidencias conclusivas que sugieran que las comunidades actuales de micromamíferos hayan sido afectadas, al menos significativamente, por eventos climáticos recientes. Se han documentado situaciones ambientales que podrían vincularse con la Anomalía Climática Medieval (ACM [siglos IX-XIII) o la PEH, en particular sequías, como aquellas que asolaron la Región Pampeana en los siglos XVIII y XIX (cf. Deschamps et al. 2003) o que generaron bajos niveles en lagos de la Patagonia (Stine y Stine 1990). Sin embargo, tales situaciones parecen haber tenido poco impacto en la dinámica específica de las comunidades de micromamíferos, al menos a juzgar por la estabilidad que se registra en los conjuntos fósiles (e. g. Pardiñas y Teta 2013). No obstante, es posible que algunos de estos pulsos climáticos hayan impactado de forma negativa, en asociación con el escenario general de disturbio. Por ejemplo, Teta et al. (2012) han conjeturado que la disminución de los parches húmedos de pastizal que indican las crónicas del siglo XVIII -por efecto de las sequías y de la predilección del ganado por estos lugares (Montoya 1984)- bien podría haber jugado un papel importante en la retracción de Bibimys torresi o Pseudoryzomys simplex, que plausiblemente ocupaban esos sectores. Contra lo expresado previamente, existen al menos algunos casos donde el efecto del clima pudo haber mostrado un papel central en los eventos de expansión y retracción distribucional. Un ejemplo de esta aseveración es el caso de la distribución holocénica del roedor anfibio Holochilus brasiliensis en latitudes medias de Argentina. Este orizomino de estirpe subtropical se habría visto favorecido por las condiciones más cálidas y benignas asociadas a la ACM y colonizado, durante el Holoceno tardío, las sierras centrales de Córdoba y los grandes ríos del norte de la Patagonia. Su posterior retracción parece vincularse con el advenimiento de las condiciones más frías y secas que caracterizaron a la PEH (Teta et al. 2005; Pardiñas y Teta 2011).

El impacto sobre los esquemas taxonómicos y zoogeográficos. Un aspecto poco explorado de la situación expuesta previamente es el potencial impacto de las extinciones regionales recientes en la elaboración de hipótesis taxonómicas y esquemas zoogeográficos. El género Bibimys constituye un buen ejemplo para discutir esta aseveración. Este akodontino tiene un área de distribución que incluye tres núcleos disjuntos, uno en el sudeste de Brasil, otro en el nordeste de Argentina y este de Paraguay y un tercero en el centro-este de Argentina, circunscripto al denominado Delta del río Paraná (Massoia 1979; Pardiñas 1996; D’Elía et al. 2005; Fig. 5). Desde una perspectiva 
histórica, la discontinuidad entre poblaciones, involucrando en todos los casos distancias de cientos de kilómetros, apoyó -explícita o tácitamente- la hipótesis de que se trataba de especies distintas (Pardiñas 1996). De esta forma se han mantenido los taxones B. labiosus (Brasil), B. chacoensis (Argentina norte y Paraguay) y B. torresi (Argentina central). Sin embargo, las evidencias moleculares y morfológicas sugieren que Bibimys sería monotípico (D'Elía et al. 2005). El registro de este roedor en distintos sitios del Holoceno tardío de la provincia de Buenos Aires (Fig. 5) y los bajos niveles de divergencia génica entre individuos de Argentina, Paraguay y Brasil no contradicen la hipótesis de una retracción reciente, sugiriendo que las discontinuidades geográficas podrían ser el resultado de extinciones regionales ocurridas en los últimos cientos de años (Pardiñas 1996, 1999a; Teta et al. 2004). De las tres especies reconocidas actualmente, la de extensión más austral es $B$. torresi, que es a su vez la de mayor tamaño, quizás reflejando cierta variación clinal. Para este taxón se han consignado capturas en ceibales, pastizales periselváticos, espadañales y pajonales altos en terrenos inundables (Massoia 1979; Pardiñas et al. 2010b). Su registro para el Holoceno tardío en sectores centrales de la Región Pampeana sugiere que también habría ocupado pastizales en terrenos más altos, incluso en áreas serranas, probablemente en la cercanía de cursos o cuerpos de agua (Pardiñas 1999a; Scheifler et al. 2012).

El caso de Pseudoryzomys simplex repite parcialmente la situación de B. torresi. Pseudoryzomys simplex es considerado como un elemento típicamente chaqueño, al menos en la porción austral de su geonemia (Voss y Myers 1991). Su distribución conocida en Argentina se restringe al Chaco Húmedo de las provincias de Formosa, Chaco y Santa Fe (Pardiñas et al. 2004). Sin embargo, hasta hace menos de 200 años este orizomino alcanzaba el sudeste de la provincia de Buenos Aires, donde incluso llegó a ser dominante en algunas comunidades (Teta et al. 2013; Fig. 5). En este contexto, no sería descabellado suponer que Pseudoryzomys habría sido una forma chacopampeana, al menos en su expresión austral. En otras palabras, su apreciación como estrictamente chaqueña, para los esquemas zoogeográficos, sería un artefacto producto de su extinción reciente en la Pampasia.

Para Patagonia, el caso de Abrothrix olivacea ilustra una situación análoga a las comentadas. Esta especie, usualmente tipificada como de la estepa patagónica y áreas boscosas adyacentes (Pearson 1995), posee varios registros aislados en el desierto del Monte (Pardiñas et al. 2003). Algunas de estas referencias tienen más de ocho décadas y fueron omitidas en la literatura posterior (e. g. Laguna del Barro, Rawson [Thomas 1929]) y otras corresponden a ejemplares depositados en colecciones, en todos los casos capturados hace más de treinta años (e. g. Choele Choel [1928], Puerto Madryn [1979]). El caso de este roedor resulta paradójico, ya que si bien por un lado desapareció del Monte austral, su frecuencia aumentó considerablemente en la Patagonia occidental (e. g. Pardiñas 1998; Pardiñas y Teta 2013). En este contexto, una hipótesis de trabajo es que ambos procesos -retracción en un ambiente y expansión en otro- hubieran estado disparados por el impacto antrópico actuando negativa y positivamente en dos contextos ambientales contrastantes. Alternativamente, su retracción y consecuente extirpación regional en el Monte podría vincularse con causales climáticas, en particular con una paulatina tendencia durante el Holoceno tardío hacia la aridificación de la Patagonia norte (Manzini et al. 2008). 
En suma, la extinción regional y reciente de algunos taxones supone ciertos sesgos que deberían ser considerados a la hora de elaborar esquemas taxonómicos y zoogeográficos. El impacto de esta omisión no ha sido mensurado, pero a juzgar por los ejemplos antes expuestos podría ser importante. Pardiñas et al. (2010a), en un análisis biogeográfico de las comunidades de roedores sigmodontinos pampeanos, han argumentado que las aproximaciones zoogeográficas efectuadas a partir de estos ensambles "artefactuales" podrían traducirse en una dilución de las barreras o en relaciones espurias entre distintas unidades. En el ejemplo de la Región Pampeana, la reputada pobreza de especies de esta unidad florística ha sido usualmente atribuida a su situación ecotonal entre dos grandes bloques faunísticos (Ringuelet 1960). Sin embargo, en realidad, bien podría responder al deterioro profundo, extensivo y reciente de los ecosistemas y la consecuente homogeneización de su biota (Rapoport 1996).
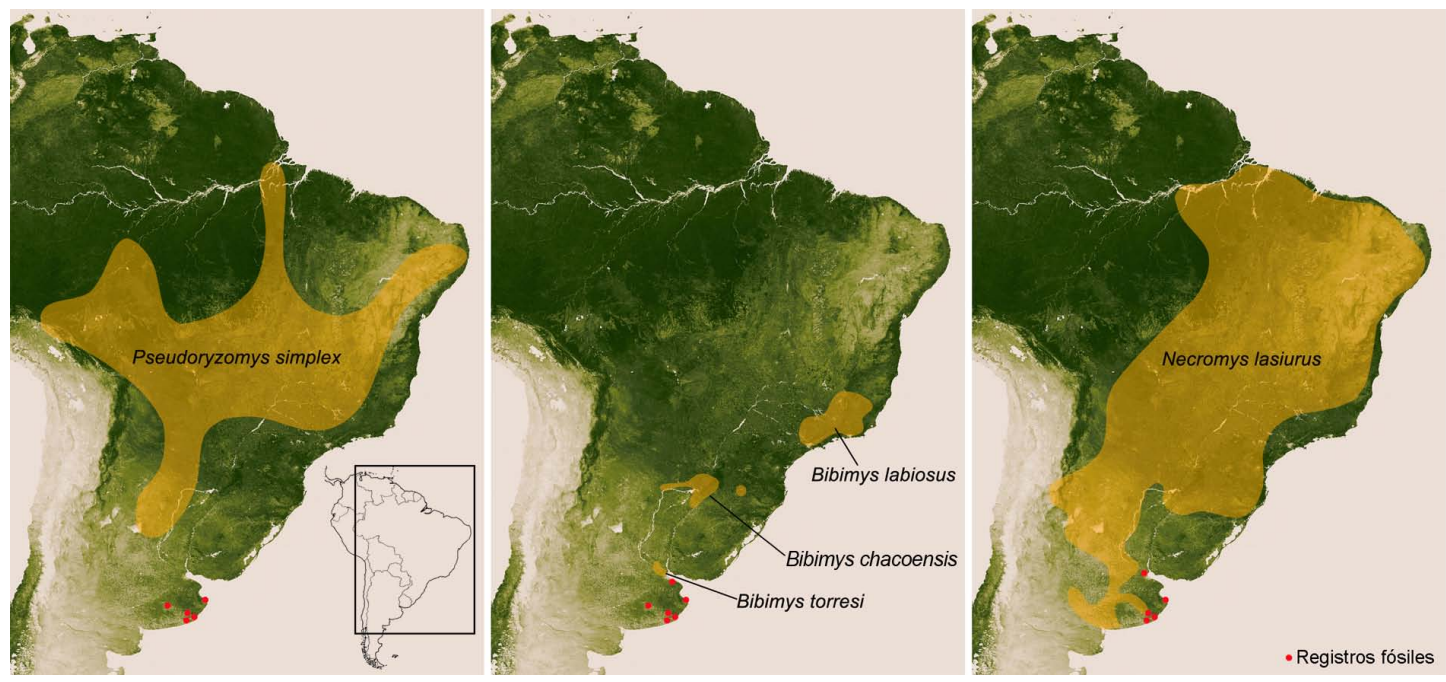

Figura 5. Distribuciones recientes (sombreado anaranjado) y fósiles durante el Holoceno tardío (puntos rojos) para algunos taxones de Sigmodontinae en América del Sur (compilado de varias fuentes, véase el texto para más detalle).

Implicancias sobre la conservación. La evidencia presentada previamente indica que incluso especies ampliamente distribuidas, cuyo estatus oficial a nivel global por parte de la Unión Internacional para la Conservación de la Naturaleza (UICN) es de Preocupación Menor (LC), han sufrido retracciones recientes (i. e. últimos siglos) que involucran de cientos a miles de kilómetros. A juzgar por el registro fósil, estos procesos parecen haber ocurrido en forma más o menos rápida, en un lapso quizás no mayor a 100-200 años. Por regla general, las estimaciones de la UICN no consideran en su evaluación más de 10 años o tres generaciones hacia el pasado, dejando de lado eventos relativamente recientes y significativos en la supervivencia de las especies. De hecho, sobre 46 especies de roedores del Cono Sur de América del Sur consideradas como LC por la UICN y con registro fósil para los últimos 500 años, 23 (50\%) han experimentado retracciones en sus áreas de distribución o reducciones drásticas de su abundancia durante el Holoceno más tardío (Tabla 1). Esta situación obliga a ser cautelosos en categorizaciones futuras, abriendo la posibilidad de considerar un segmento más amplio de la historia de los taxones, que destaque sus respuestas previas frente a cambios ambientales excepcionales.

Amori et al. (2013) han sugerido que en América del Sur las especies de roedores más amenazadas son especialistas de pastizales, matorrales o selvas. El registro fósil 
de la Región Pampeana es consistente con esta hipótesis, desde que en este bioma se han perdido hasta seis especies en algunas localidades en los últimos 0,5 ka (e. g. Pardiñas 1999a, 2000; Teta et al. 2013; Fig. 1, 5 y 6). En suma, la situación destacada por Amori et al. (2013) y este trabajo plantean la necesidad de enfocar las estrategias de conservación hacia áreas de pastizal y matorral o a los mosaicos entre estos ambientes y áreas forestadas (véase también Emmons 2009).

Para muchas especies supuestamente extintas, se requieren nuevos trabajos de campo que permitan confirmar o descartar esa hipótesis. Además, son necesarias aproximaciones novedosas que contribuyan a despejar la incertidumbre taxonómica que rodea a algunos de estos taxones. Por ejemplo, Galea tixiensis se erige como una especie extinta propia del sistema serrano de Tandilia, en el sudeste de la provincia de Buenos Aires (Quintana 2001b). Aparentemente, este cávido habría mantenido poblaciones importantes durante todo el Holoceno, a juzgar por la secuencia del sitio Cueva Tixi, para declinar y desaparecer con un último registro hace $\sim 170$ ar AP. En su lugar hoy día habita la región -y extensivamente en la Argentina- Galea leucoblephara. Sin embargo, son necesarios nuevos estudios que permitan, por un lado, discutir si los rasgos morfológicos propuestos como diagnósticos de G. tixiensis son definitorios de estatus específico o si son simplemente variaciones dentro de G. leucoblephara. Por el otro, también sería conveniente un relevamiento extensivo de las sierras de Tandilia destinado a la detección de potenciales poblaciones relictuales de G. tixiensis. Una situación similar se plantea con respecto a Cryptonanus ignutus, que para algunos autores podría constituir un sinónimo de C. chacoensis (Voss et al. 2005).

La extinción biológica de una especie representa el punto final de una larga secuencia de declinaciones poblacionales y extinciones locales (Collen et al. 2011). Por lo tanto, comprender las causas que guían estos procesos constituye un paso imprescindible en la elaboración de planes adecuados de manejo y conservación. Por ejemplo, desconocemos cuantas especies están pagando todavía su "deuda de extinción" (e. g. Jackson y Sax 2010; Kuussaari et al. 2010; Hylander y Ehrlén 2013), especialmente en ambientes que han sido profundamente alterados. Del mismo modo, tampoco sabemos cómo pudo haber influido en este proceso la regulación "desde arriba" que ejercían depredadores mayores, como el puma o el yaguareté, sobre los depredadores medianos o menores, como zorros o zorrinos. El relajamiento de los niveles medios de las cadenas tróficas -ante la ausencia de grandes depredadores- pudo haber influido negativamente sobre las poblaciones de roedores, tal como ha sido demostrado a escala ecológica (e. g. Johnson et al. 2006 para Australia). A esto debe sumarse la presencia de perros cimarrones (Canis familiaris), cuya abundancia durante los siglos XVIII y XIX fue destacada por distintos autores (e. g. Gibson 1908; MacCann 1939) y que seguramente hayan ejercido un efecto negativo sobre la fauna. La perspectiva que ofrecen los estudios de conjuntos fósiles y arqueológicos resulta promisoria en este sentido, aportando evidencias en un rango temporal más extenso que los usualmente considerados (e. g. Terry 2010). El hallazgo y estudio de nuevos yacimientos es indispensable para seguir sumando elementos, a la vez que es necesario un conocimiento más completo de las comunidades actuales.

Estudios realizados en Australia sugieren que numerosas especies de micromamíferos no sólo han sufrido declinaciones severas en su distribución y abundancia, sino que también se habría contraído el nicho que ocupaban. En efecto, hay taxones que 
en la etapa previa a la llegada de los europeos habitaban una mayor diversidad de ambientes y regiones (Bilney et al. 2010). Al menos conjeturalmente, no se puede dejar de pensar en cierto paralelismo entre el ejemplo australiano y la situación de especies como P. simplex o B. torresi, que desaparecieron por completo de los pastizales de la Pampasia. Sin embargo, este es otro aspecto que permanece inexplorado y a la espera de aproximaciones detalladas.

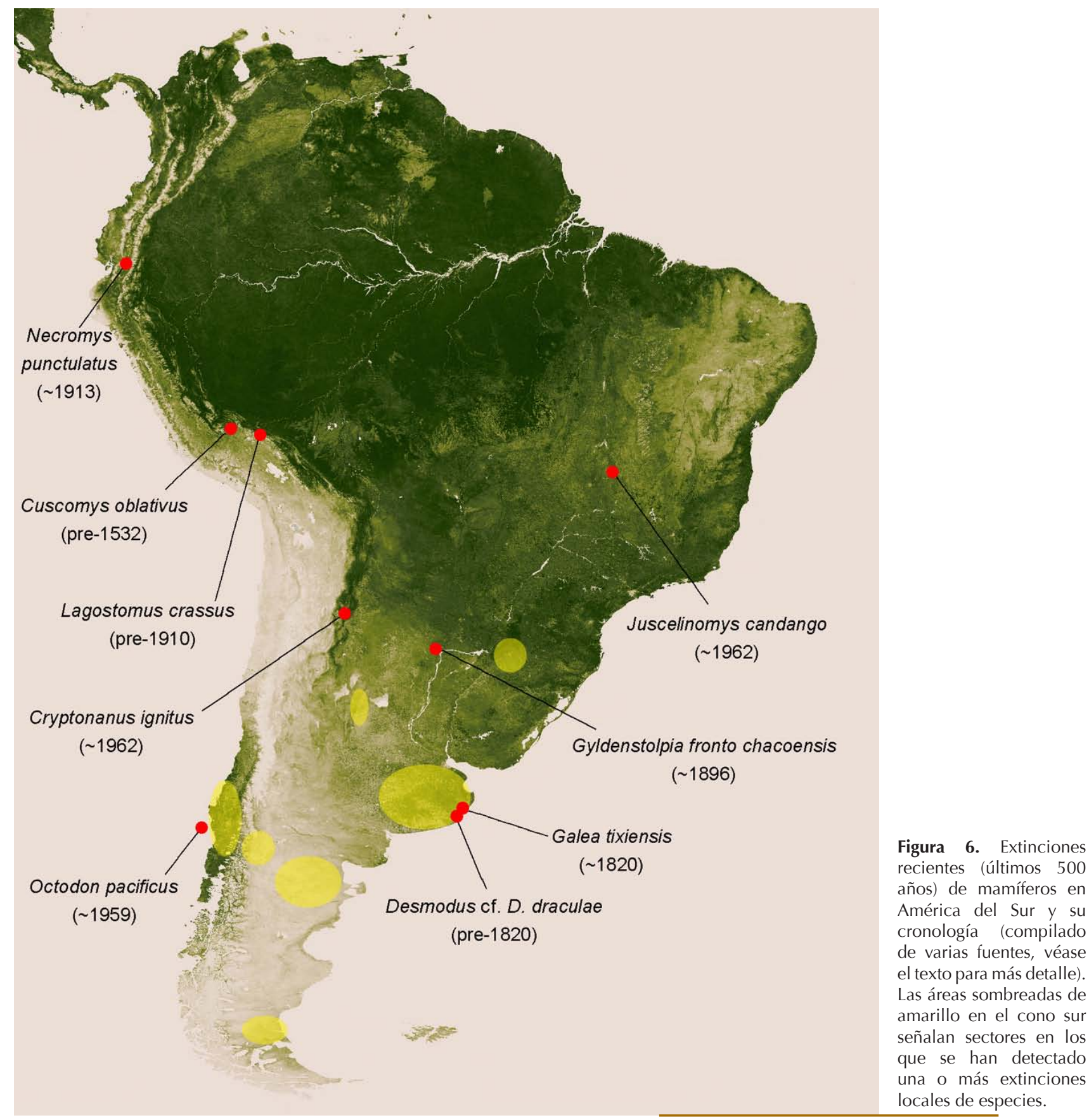

Declinación de micromamíferos en el Hemisferio Sur. La declinación y extinción de especies durante el segmento más tardío del Holoceno es un fenómeno generalizado en el Hemisferio Sur. En Sudáfrica, Avery (1992) vinculó la dominancia de especies 
oportunistas y una declinación de la diversidad con el impacto de actividades agropecuarias, aunque reconociendo diferencias locales y una mayor o menor influencia del clima con variación regional. De acuerdo con esta autora, la influencia antrópica comienza tempranamente, a través del establecimiento de poblaciones de pastores en áreas abiertas de pastizal hacia 1-2 ka y recrudece en los últimos 100-150 años, con la agricultura y ganadería extensiva realizada por los colonos europeos. Para Avery (1987, 1992, 1997), las retracciones recientes (<200 años) y significativas en la distribución de algunos taxones se vincularían con ciertas tendencias climáticas y/o la introducción de ganado ovino y la consecuente reducción de los parches de pastizal. Avery (1981) sugiere incluso que la distribución fragmentaria de algunas especies podría relacionarse con el efecto reciente del sobrepastoreo. A su vez, indica que el establecimiento regional de granjeros y pastores favoreció la expansión de formas comensales como aquellas de los géneros Mus y Rattus.

En Chile, Simonetti (1994) planteó una situación similar, relacionando la desaparición de algunas especies de octodóntidos con la extracción de leña realizada por las comunidades aborígenes hacia $1.7 \mathrm{ka}$. Otros empobrecimientos menos explorados -pero igualmente profundos- parecen haber tenido lugar en sectores norandinos de Ecuador. Los cricétidos del Holoceno tardío en la localidad de La Calera componían una comunidad diversa incluyendo un taxón de afinidades inciertas, Copemyodon, hoy día aparentemente extinto. Los trampeos en las mismas áreas revelan ensambles pauperizados, quizás en consonancia con un largo proceso de impacto antrópico prey posthispánico (Fejfar et al. 1993, 1996). Los ejemplos mencionados sugieren que si bien el impacto antrópico ha sido más acusado en los últimos 500 años, ya en etapas anteriores se podría haber hecho sentir la influencia de la presencia humana, al menos localmente. En general, la naturaleza y extensión del impacto humano prehistórico (> 500 años) sobre las comunidades contemporáneas ha sido ignorado, quizá por no tener la magnitud de los cambios ocurridos en los últimos cientos de años.

En Australia, por su condición insular, la situación ha sido más crítica. Casi la mitad de los mamíferos que se extinguieron durante los últimos 200 años eran endémicos de esa isla. En efecto, de 250 mamíferos nativos, al menos 24 pueden considerarse extintos y otros 26 se sostienen en áreas que representan no más del 20\% de su distribución original (Short y Smith 1994). En su mayoría, se trata de roedores y marsupiales de tamaño pequeño a mediano, con pesos entre 30 y 5,500 g. La extirpación regional de especies ha sido más acusada en el interior árido, en áreas transformadas extensivamente al cultivo de cereales y la ganadería ovina (Short y Smith 1994). En las áreas forestadas del sudeste australiano, de 28 especies detectadas en muestras fósiles (0.4-0.1 ka) generadas por la lechuza Tyto tenebricosa, sólo 10 se registran en la dieta actual de esta misma rapaz (Bilney et al. 2010). La introducción de depredadores exóticos de tamaño mediano parece haber jugado un papel central en estos procesos, por su fuerte presión sobre la comunidades de pequeños mamíferos (Jonson et al. 2006). A esto se sumaría la alteración de los regímenes naturales de fuego, especialmente en extensión e intensidad.

\section{Conclusiones}

Esta contribución revela que durante los últimos 500 años se produjeron cambios significativos en los ensambles de micromamíferos del sur de América del Sur. Estos 
cambios han incluido desde el aumento, muchas veces dramático, de especies oportunistas, hasta la extinción regional de otros taxones. Sin embargo, las causales de estos procesos -de componente antrópico mayoritario- no resultan por el momento claras ni, quizás, dependientes de un único factor. Del mismo modo, tampoco tenemos un conocimiento detallado de la cronología de estos sucesos, más allá de coincidir laxamente en que se habrían producido en forma posterior a la llegada de los europeos.

Por otro lado, varias especies que presentan marcadas retracciones parecen estar respondiendo a un proceso de cambio climático, en particular en Patagonia, a escala milenaria y que habría favorecido la expansión de elementos orientales en detrimento de aquellos occidentales (e. g. Pardiñas et al. 2012). Superpuesto a este proceso se verifican situaciones más puntuales de impacto antrópico de gran magnitud (i. e. sobrepastoreo, desertificación), que parecen haber disparado reestructuraciones locales profundas en los ensambles, con reducciones significativas de la riqueza y diversidad de especies.

En suma, tanto en el caso de América del Sur como de otras áreas del Hemisferio Sur, las comunidades de micromamíferos parecen haber sido profundamente afectadas en su estructura en la etapa inmediatamente posterior a la llegada de los europeos. Si bien existen esperables diferencias locales, las actividades que parecen haber contribuido más con estos cambios son la ganadería extensiva, los cambios en los regímenes de fuego y la transformación de los ambientes naturales en agroecosistemas. Para algunos taxones, nuestro entendimiento de su verdadero nicho y potencial ecológico es todavía limitado. Fenómenos como los indicados más arriba (e. g. declinaciones, extinciones locales) podrían resultar en disrupciones severas de las funciones ecosistémicas, cuyas consecuencias a gran escala son desconocidas. En este sentido, la necesidad de establecer planes de manejo y conservación de pequeños mamíferos resulta crucial si lo que se pretende es mantener las funciones provistas por estos animales.

Esta contribución es el resultado de más de una década de estudios, que incluyeron la participación, tanto en el campo como en el gabinete, de numerosas personas. Entre aquellas que han estado más largamente vinculadas con las tareas deseamos agradecer a A. Bernardis, D. Podestá, D. Udrizar-Sauthier y P. Wallace. Hacemos extensivo nuestro reconocimiento a R. Owen (y a través de él, a S. T. Álvarez), por invitarnos a participar de este volumen especial y por sus comentarios, que junto con los de tres evaluadores anónimos, contribuyeron a mejorar este manuscrito. El soporte económico fue provisto por los proyectos de investigación PIP (CONICET) 6179, Grant 7813-05 (National Geographic Society) y PICT 2008, \#0547 (ANPCyT).

Aagesen, D. 2000. Crisis and conservation at the end of the world: sheep ranching in Argentine Patagonia. Enviromental Conservation 27:208-215.

Allen, J. A. 1905. I. The Mammalia of Southern Patagonia. Reports of the PrincetonUniversity Expeditions to Patagonia, 1896-1899. Volume 3, Zoölogy, Princeton, EE.UU. 
Amori, G., F. Chiozza, B. D. Patterson, C. Rondinini, J. Schipper y L. Luiselli. 2013. Species richness and distribution of Neotropical rodents, with conservation implications. Mammalia 77:1-19.

Andrews, P. 1990. Owls, caves and fossils. Predation, Preservation, and Accumulation of small mammal bones in caves, with an analysis of the PleistoceneCave faunas from Westbury-sub- Mendip, Somerset, UK. Chicago, University of Chicago Press. Chicago, EE.UU.

Ares, J., A. M. Beeskow, M. Bertiller, M. Rostagno, M. Irusarri, J. Anchorena, G. Defosse, Y C. Merino. 1995. Structural and Dynamic characteristics of overgrazed lands of northern Patagonia, Argentina. Pp. 149-175 en Managed Grasslands (Breymeyer, A., ed.). Elsevier Science Publishers B. V. Amsterdam, Holanda.

Avery, D. M. 1981. Holocene micromammalian faunas from the northern Cape Province, South Africa. South African Journal of Science 77:265-273.

Avery, D. M. 1982. Micromammal as palaeoenvironmental indicators and an interpretation of the late Quaternary in the southern Cape Province, South Africa. Annals of the South African Museum 85:183-374.

Averr, D. M. 1987. Micromammalian evidence for natural vegetation and the introduction of farming during the Holocene in the Magaliesberg, Transvaal. South African Journal of Science 83:221-225.

Avery, D. M. 1992. Micromammasl and the environment of early pastoralists at Spoeg river, western Cape Province, South Africa. South African Archaeological Bulletin 47:116-121

Avery, D. M. 1997. Micromammals and the Holocene environment of Rose Cottage Cave. South African Journal of Science 93:445-448.

Barnosky, A. D., Y E. L. Lindsey. 2010. Timing of Quaternary megafaunal extinction in South America in relation to human arrival and climate change. Quaternary International 217:10-29.

Belloce, M. I. 2000. A review of the trophic ecology of the barn owl in Argentina. Journal of Raptor Research 34:108-119.

Bılenca, D., y F. Krávetz. 1995. Patrones de abundancia relativa en ensambles de pequeños roedores de la región pampeana. Ecología Austral 5:21-30.

Bilenca, D., M. Codesido, C. González Fischer, l. Pérez Carusi, E. Zufiaurre, y A. Abba. 2012.Impactos de la transformación agropecuaria sobre la biodiversidad en la provincia de Buenos Aires. Revista del Museo Argentino de Ciencias Naturales n.s. 14:189-198.

Bilney, R. J., R. Coоke, y J. G. White. 2010. Underestimated and severe: Small mammal decline from the forests of south-eastern Australia since European settlement, as revealed by a top-order predator. Biological Conservation 143:52-59.

Bertiller, M. B., y A. Bisigato. 1998. Vegetation dynamics under grazing disturbance. The state-and-transition model for the Patagonian steppes. Pp. 191-199 en Ecosistemas patagónicos (Oesterheld, M., M. R. Aguiar, y J. M. Paruelo, eds.). Ecología Austral 8:75-308.

Bó, M. S., A. V. Baladrón, y L. M.Bıondi. 2007. Ecología trófica de Falconiformes y Strigifores: tiempo de síntesis. Hornero 22:97-115. 
Borrero, L. A. 2007. Extinction of Pleistocene megamammals in South America: The lost evidence. Quaternary International 185:69-74.

Brown, J. H. 1984. On the relationship between abundance and distribution of species. American Naturalist 124: 255-279.

Cavia, R., G. R. Cueto, y O. V. Suárez. 2008. Changes in rodent communities according to the landscape structure in an urban ecosystem. Landscape and Urban Planning 90:11-19.

Céspedes del Castillo, G. 2009. América Hispánica, 1492-1898. Marcial Pons, Ediciones de Historia. Barcelona, España.

Chan, Y. L., E. A. Lacey, O. P. Pearson, y E. A. Hadly. 2005. Ancient DNA reveals Holocene loss of genetic diversity in a South American rodent. Biology Letters 1:423-426.

Chan, Y. L., y E. A. Hadiy. 2011. Genetic variation over 10,000 years in Ctenomys: comparative phylochronology provides a temporal perspective on rarity, environmental change and demography. Molecular Ecology 20:4592-4605.

Chiappero, M. B., M. S. Sabattini, A. Blanco, G. E. Calderón, y C. N. Gardenal. 2002a. Gene flow among Calomys musculinus (Rodentia, Muridae) populations in Argentina. Genetica 114:63-72.

Chiappero, M. B., A. Blanco, G. E. Calderón, M. S. Sabattini, y C. N. Gardenal. 2002b. Genetic structure of populations of Calomys laucha (Muridae, Sigmodontinae) from Central Argentina. Biochemical Systematics and Ecology 30:1023-1036.

Cione, A. L., E. P. Tonni, y L. Soibezzon. 2003. The Broken Zig-Zag: Late Cenozoic large mammal and tortoise extinction in South America. Revista del Museo Argentino de Ciencias Naturales 5:1-19.

Codesido, M., C. Gonzalez-Fisher, y D. N. Bilenca. 2011. Distributional changes of landbird species inagroecosystems of Central Argentina. The Condor113:266-273.

Codesido, M., C. Gonzalez-Fisher, y D. N. Bilenca. 2012. Agricultural land-use, avian nesting and rarity in the Pampas of central Argentina. Emu 112:46-54.

Collen, B., L. Mcrae, S. Deinet, A. De Palma, T. Carranza, N. Cooper, J. Loh, y J. E. M. Ballute. 2011. Predicting how populations decline to extintion. Philosophical Transactions of The Royal Society B 366:2577-2586.

Comparatore, V. M., M. M. Martínez, A. I. Vasallo, M. Barg, y J. P. Isacch. 1996. Abundancia y relaciones con el hábitat de aves y mamíferos en pastizales de Paspalum quadrifarium (Paja Colorada) manejados con fuego (Prov. de Buenos Aires, Argentina). Interciencia 21:228-237.

D'Elía, G., U. F. J. Pardiñas, y P. Myers.2005. An introduction to the genus Bibimys (Rodentia: Sigmodontinae): Phylogenetic position and alpha taxonomy. Pp. 211246 en Mammalian diversification: from Chromosomes to Phylogeography (A Celebration of the Career of James L. Patton) (Lacey, E., y P. Myers, eds.). University of California Publication in Zoology. Berkeley, EE.UU.

Deschamps, J., O. Otero, y E. P. Tonni.2003. Cambio climático en la pampa bonaerense: las precipitaciones desde los siglos XVIII al XX:1-18. Documento de Trabajo $N^{\circ}$ 109, Universidad de Belgrano. Disponible en la red: http://www.ub.edu.ar/ investigaciones/dt_nuevos/109_deschamps.pdf 
de Tommaso, D. C., A. E. Formoso, P. Teta, D. E. Udrizar Sauthier. y U. F. J. Pardiñas. En prensa. Distribución geográfica de Calomys musculinus (Rodentia, Sigmodontinae) en Patagonia. Mastozoología Neotropical.

Devictor, V., R. Julliard, y F. Jiguet. 2008. Distribution of specialist and generalist species along spatial gradients of habitat disturbance and fragmentation. Oikos 117:507514.

Dı Gıсомо, A. S., y A. G. Dı Gıасомо. 2004. Extinción, historia natural y conservación de las Poblaciones del Yetapá de Collar (Alectrurus risora) en la Argentina. Ornitología Neotropical 15:145-157.

Emmons L. H. 2009. Long-term variation in small mammal abundance in forest and savanna of Bolivian Cerrado. Biotropica 41:493-502.

Fejfar, O., A. Blasetti, G. Calderoni, M. Coltorti, G. Ficcarelli, F. Masini, L. Rook, y D. Torre. 1993. New fossil finds of Cricetids in Northern Ecuador. Documents des Laboratoires de Géologie, Lyon 125:151-167.

Fejfar, O., G. Ficcarelli, C. Mezzabotta, M. Moreno Espinosa, L. Rook, y D. Torre. 1996. First record of a Copemyne-Peromyscine form in South America. Hypotheses on its ancestry in Palearctic. Acta Zoologica Cracoviensia 38:137-145.

Fernández, F. J. 2012. Microvertebrados del Holoceno de sitios arqueológicos en el sur de Mendoza (República Argentina): Aspectos tafonómicos y sus implicancias en la subsistencia humana. Tesis doctoral. Facultad de Ciencias Naturales y Museo. Universidad Nacional de la Plata. Buenos Aires, Argentina.

Formoso, A. E. 2013. Ensambles de micromamíferos y variables ambientales en Patagonia continental extra-andina argentina. Tesis doctoral. Facultad de Ciencias Naturales y Museo. Universidad Nacional de la Plata. Buenos Aires, Argentina.

Fraga, R. M. 2003. Distribution, natural history and conservation of the Black-andwhite Monjita (Heteroxolmis dominicana) in Argentina, a species vulnerable to extinction. Ornitología Neotropical 14:145-156.

Galeano, E. 1971. Las venas abiertas de América Latina. Editorial Siglo XXI. Buenos Aires, Argentina.

Galliarı, C. A, W. D. Berman, y F. J. Goin. 1991. Mamíferos. Situación Ambiental de la provincia de Buenos Aires. Recursos y rasgos naturales en la evaluación ambiental. Comisión Investigaciones Científicas 1:1-35. La Plata, Argentina.

Galliarı, C. A., y U. F. J. Pardiñas. 2000. Taxonomy and distribution of the sigmodontine rodents of genus Necromys in central Argentina and Uruguay. Acta Theriologica 45:211-232.

García Esponda, C. M., L. J. M. de Santis, J. I. Noriega, G. O. Pagnoni, G. J. Moreira, y M. N.Bertellotti. 1998. The diet of Tyto alba (Strigiformes: Tytonidae) in the lower Chubut valley river (Argentina). Neotrópica 44:57-63.

Gibertı, H. C. E. 1986. Historia económica de la ganadería argentina. Biblioteca argentina de historia y política. Hyspamérica Ediciones Argentina. Buenos Aires, Argentina.

Gıвson, H. 1908. La Evolución Ganadera.Pp. 57-99 en Censo Agropecuario Nacional, 1908, Tomo III. Buenos Aires, Argentina. 
Ghersa, C. M., M. A. Martinez-Gherza, y R. J. C. Leon. 1998. Cambios en el paisaje pampeano y sus efectos sobre los sistemas de soporte de la vida. Pp. 38-71 en Hacia una agricultura productiva y sostenible en la pampa (Solbrig, O. T., y L. Vainesman, comp.). Harvard University Press y Consejo Profesional de Ingeniería Agronómica. Buenos Aires, Argentina.

González Fischer, C. M., G. Baldi, M. Codesido, y D. Bilenca. 2012. Seasonal variations in small mammal-landscape associations in temperate agroecosystems: a study case in Buenos Aires province, central Argentina. Mammalia 76:339-406.

Gray, J. S. 1989. Effects of environmental stress on species rich assemblages. Biological Journal of the Linnean Society 37:19-32.

Guthmann, N., M. lozada, J. A. Monjeau, y K. M. Heinemann. 1997. Population dynamics of five sigmodontine rodents of northwestern Patagonia. Acta Theriologica 42:43152.

Haynes, G. 2009. American Megafaunal Extinctions at the End of the Pleistocene. Springer, Dordecht, The Netherlands.

Hercolini, C.2007. Efectos de la urbanización sobre las comunidades de pequeños roedores del Área Metropolitana de Buenos Aires, Argentina. Tesis de Licenciatura. Facultad de Ciencias Exactas y Naturales, Universidad de Buenos Aires. Buenos Aires, Argentina.

Hylander, K., y J. Ehrlén. 2013. The mechanisms causing extinction debts. Trends in Ecology and Evolution 28:341-346.

JaCkSON, S. T., Y D. F. SAx. 2010. Balancing biodiversity in a changing environment: extinction debt, immigration credit and species turnover. Trends in Ecology and Evolution 24:153-160.

Johnson, C. N., J. L. IsAaC, y D. O. Fisher. 2007. Rarity of a top predator triggers continentwide collapse of mammal prey: dingoes and marsupials in Australia. Proceedings of the Royal Society B 274:341-346.

Kuussaari, M., R. Bommarco, R. K. Heikinen, A. Helm, J. Krauss, R. Lindborg, E. Ockinger, M. Partel, J. Pino, F. Roda, C. Stefanescu, T. Teder, M. Zobel, y I. Steffan-Dewenter. 2010. Extinction debt: a challenge for biodiversity conservation. Trends in Ecology and Evolution 24:564-571.

León, R. J. C., y M. R. Aguiar. 1985. El deterioro por uso pasturil en estepas herbáceas patagónicas. Phytocoenología 13:181-196.

León, R. J. C., D. Bran, M. Collantes, J. M. Paruelo y A. Soriano. 1998. Grandes unidades de vegetación de la Patagonia extraandina. Pp. 75-308 en Ecosistemas patagónicos (Oesterheld, M., M.R. Aguiar, y J.M. Paruelo, eds.). Ecología Austral8.

Lomolino, M. V., y R. Channell. 1995. Splendid isolation: patterns of geographic range collapse in endangered mammals. Journal of Mammalogy 76:335-347.

MacCanN, W. 1939. Viaje a caballos por las provincias argentinas. Dimensión Argentina, Solar/Hacette.

MacPhee, R. D. E., C. Flemming, y D. P. Lunde. 1999. "Last occurrence" of the Antillean insectivoran Nesophontes: new radiometric dates and their interpretation. American Musseum Novitates 3261:1-20. 
MacPhee, R. D. E., y C. Flemming. 1999. Requiem Æternam. The last five hundred years of mammalian. Species extinctions. Pp. 333-371 en Extinctions in near time (MacPhee, R. D. E., ed.). Kluwer Academic/Plenum Publishers. New York, EE.UU.

Manzini, M. V., A. R. Prieto, M. M. Paez, y F. Schabitz. 2008. Late quaternary vegetation and climate of Patagonia. Pp. 351-368 en The Late Cenozoic of Patagonia and Tierra del Fuego (Rabassa, J. ed.). Developments in Quaternary Sciences, 11. Elsevier. Amsterdam, The Netherlands.

Marconi, P. N. 1988. Efecto de las perturbaciones intensas sobre la estructura de las comunidades de roedores. Tesis Doctoral. Facultad de Ciencias Exactas y Naturales, Universidad de Buenos Aires.Buenos Aires,Argentina.

Massoia, E. 1979. Descripción de un género y especie nuevos: Bibimys torresi (Mammalia- Rodentia- Cricetidae- Sigmodontinae- Scapteromyini). Historia Natural 2:57-63.

McKınNeY, M. L., Y J. L. Lockwood. 1999. Biotic homogenization: a few winners replacing many losers in the next mass extinction. Trends in Ecology and Evolution 14: $450 c 453$.

Medan, D., J. P. Torretta, K. Hodara, E. B. de la Fuente, y N. H. Montaldo. 2011. Effects of agriculture expansion and intensification on the vertebrate and invertebrate diversity in the Pampas of Argentina. Biodiversity and Conservation 20:30773100.

Mills, J. N., B. A. Eluis, K. T. McKee, J. I. Maiztegui, y J. E. Childs. 1992. Reproductive characteristics of rodent assemblages in cultivated regions of central Argentina. Journal of Mammalogy 73:515-526.

Monjeau, J. A. 1989. Ecología y distribución geográfica de los pequeños mamíferos del Parque Nacional Nahuel Huapi y áreas adyacentes. Tesis Doctoral, Facultad de Ciencias Naturales y Museo, Universidad Nacional de La Plata. La Plata, Argentina.

Montora, A. J. 1984. Cómo evolucionó la ganadería en la época del virreinato. Contribución de Manuel José de Lavardén a su desarrollo y mejoramiento. Editorial Plus Ultra. Buenos Aires, Argentina.

Morello, J., G. D. Buzal, C. A. Baxendale, A. F. Rodríguez, S. D. Matteucci, R. E. Godagnone, Y R. R. CASAS. 2000. Urbanización y consumo de alta fertilidad. El caso del Gran Buenos Aires en la Pampa Ondulada argentina. Ciencia Hoy 10:50-61.

OjedA, R. A. 1989. Small mammal responses to fire in the Monte desert, Argentina. Journal of Mammalogy 70:416-420.

Ortiz, P. E. 2001. Roedores del Pleistoceno superior del Valle de Tafí (provincia de Tucumán). Implicancias paleoambientales y paleobiogeográficas. Tesis Doctoral, Universidad Nacional de Tucumán. San Miguel de Tucumán, Argentina.

Ortiz, P. E., J. P. Jayat, y U. F. J. Pardiñas. 2011. Fossil sigmodontine rodents of Northwestern Argentina: taxonomy and paleoenvironmental meaning. Pp. 301316 en Cenozoic Geology of the Central Andes of Argentina (Salfity, J. A., y R. A. Marquillas, eds.), SCS Publisher. Salta, Argentina. 
Osorıo, M. V. 2003. Respuesta de micromamíferos al fuego en Península Valdés, analizada a través de la dieta de Speotyto cunicularia (Aves: Strigidae). Tesis de Licenciatura, Facultad de Ciencias Naturales, Universidad Nacional de la Patagonia San Juan Bosco. Puerto Madryn, Argentina.

Pardiñas, U. F. J. 1995. Los roedores cricétidos. Pp. 229-256 en: Evolución climática y biológica de los últimos cinco millones de años en la región pampeana. Un ensayo de correlación con el Mediterráneo Occidental (Alberdi, M. T., G. Leone, y E. P. Tonni, eds.). Monografías Museo Nacional de Ciencias Naturales, CSIC. Madrid, España.

Pardiñas, U. F. J. 1996. El registro fósil de Bibimys Massoia, 1979 (Rodentia). Consideraciones sobre los Scapteromyini (Cricetidae, Sigmodontinae) y su distribución durante el Plioceno-Holoceno en la región pampeana. Mastozoología Neotropical 3:15-38.

Pardiñas, U. F. J. 1998. Roedores holocénicos del sitio Cerro Casa de Piedra 5 (Santa Cruz, Argentina): tafonomía y paleoambientes. Palimpsesto 5:66-90.

Pardiñas, U. F. J. 1999a. Los roedores muroideos del pleistoceno tardio-holoceno en la región pampeana (sector este) y Patagonia (República Argentina): aspectos taxonómicos, importancia bioestratigráfica y significación paleoambiental. Tesis Doctoral. Facultad de Ciencias Naturales y Museo, Universidad Nacional de La Plata. Buenos Aires, Argentina.

Pardiñas, U. F. J. 1999b. Fossil murids: Taxonomy, Paleoecology, and Paleoenvironments. Quaternary of South America and Antarctic Peninsula 12:225-254.

Pardiñas, U. F. J. 2000. Tafonomía de microvertebrados en yacimientos arqueológicos de Patagonia (Argentina). Arqueología 9:265-340.

Pardiñas, U. F. J., y S. Cirignoli. 2002.Bibliografía comentada sobre los análisis de egagrópilas de aves rapaces en Argentina. Ornitologia Neotropical 13:31-59.

Pardiñas, U. F. J., y P. Teta. 2011. Fossil history of the marsh rats of the genus Holochilus and Lundomys (Cricetidae, Sigmodontinae) in southern South America. Estudios Geológicos 67:111-129.

Pardiñas, U. F. J., y P. Teta. 2013. Holocene stability and recent dramatic changes in micromammalian communities of northwestern Patagonia. Quaternary International 305:127-140.

Pardiñas, U. F. J., y E. P. Tonnı. 2000. A giant vampire (Mammalia, Chiroptera) in the Late Holocene from the Argentinean pampas: paleoenvironmental significance. Palaeo 160:213-221.

Pardiñas, U. F. J., G. Moreira, C. García-Esponda, y L. J. M. De Santis. 2000. Deterioro ambiental y micromamíferos durante el Holoceno en el nordeste de la estepa patagónica (Argentina). Revista Chilena de Historia Natural 73:9-21.

Pardiñas, U. F. J., P. Teta, S. Cirignol, y D. Podestá. 2003. Micromamíferos (Didelphimorphia y Rodentia) de norpatagonia extra andina, Argentina: taxonomía alfa y biogeografía. Mastozoología Neotropical 10:69-113.

Pardiñas, U. F. J., S. Cirignoli, y C. Galliari.2004. Distribution of Pseudoryzomys simplex (Rodentia: Cricetidae) in Argentina. Mastozoología Neotropical 11:105-108. 
Pardiñas, U. F. J., D. E. Udrizar Sauthier, A. Andrade, y P. Teta. 2005. Paleoambientesdel Holoceno tardío en Patagonia norte extra-andina (Argentina): los micromamíferos comoevidencia. Pp. 8 en: Actas del XVI Congreso Geológico Argentino. CD-ROM, Artículo No804. (Cabaleri, N., C. A. Cingolani, E. Linares, M. G. López de Luchi, H. A. Ostera, y H. O.Panarello, eds.).La Plata. Buenos Aires, Argentina.

Pardiñas, U. F. J., G. D’Elía, y P. Teta. 2008. Una introducción a los mayores sigmodontinos vivientes: revisión de Kunsia Hershkovitz, 1966 y descripción de un nuevo género (Rodentia: Cricetidae). Arquivos do Museu Nacional, Rio de Janeiro 66:509-594.

Pardiñas, U. F. J., P. teta, y D. N. Bilenca.2010a. Roedores Sigmodontinos de la Región Pampeana: una introducción zoogeográfica. Pp. 37-57en Biología y ecología de pequeños roedores en la Región Pampeana de Argentina: enfoques y perspectivas (Polop J. J., y M. Busch, eds.). Universidad Nacional de Córdoba. Córdoba, Argentina.

Pardiñas, U. F. J., P. Teta, y G. D’Elía. 2010b. Roedores sigmodontinos de la región pampeana: historia evolutiva, sistemática y taxonomía. Pp. 9-36 en Biología y ecología de pequeños roedores en la región pampeana de Argentina: enfoques y perspectivas (Polop, J. J., y M. Busch, eds). Universidad Nacional de Córdoba. Córdoba, Argentina.

Pardiñas, U. F. J., P. Teta., G. D’Elía, y E. P. Lessa. 2011. The evolutionary history of sigmodontine rodents in Patagonia and Tierra del Fuego. Biological Journal of the Linnean Society 103:495-513.

Pardiñas, U. F. J., D. E. Udrizar Sauthier, y P. Teta. 2012. Micromammal diversity loss in central-eastern Patagonia over the last 400 years. Journal of Arid Environments 85:71-75.

Pearson, O. P. 1987. Mice and the postglacial history of the traful valley of Argentina. Journal of Mammalogy 68:469-478.

Pearson, O. P. 1995. Annotated keys for identifying small mammals living in or near Nahuel Huapi National Park or Lanín National Park, southern Argentina. Mastozoología Neotropical 2:99-148.

Pearson, O. P., y A. K. Pearson. 1993. La fauna de mamíferos pequeños cerca de Cueva Traful I, Argentina: pasado y presente. Præhistoria 1:73-89.

Pearson, O. P., S. Martin, y J. Bellati. 1987. Demography and reproduction of the silky desert mouse (Eligmodontia) in Argentina. Fieldiana, Zoology 39:433-446.

Perelman, S. B., R. J. C. León, y J. P. Bussacca. 1997. Floristic changes related to grazing intensity in a Patagonian shrub steppe. Ecography 20:400-406.

Quintana, C. 2001a. Galea (Rodentia, Caviidae) del Pleistoceno Superior y Holoceno de las sierras de Tandilia Oriental, Provincia de Buenos Aires, Argentina. Ameghiniana 38:399-408.

Quintana, C. 2001b. Composición y cambios en la secuencia faunística. Pp. 37-64 en Cueva Tixi: cazadores y recolectores de las sierras de Tandilia Oriental (Mazzanti, D., y C. Quintana, eds.). 1 Geología, Paleontología y Zooarqueología. Publicación Especial del Laboratorio de Arqueología. Universidad Nacional de Mar del Plata. Buenos Aires, Argentina.

RAPOPORT, E. H. 1996. The flora of Buenos Aires: low richness or mass extinction? International Journal of Ecology and Environmental Sciences 22:217-242. 
Rebane, K. 2002. The effects of historic climatic change and anthopogenic disturbance on rodent communities in Patagonia, Argentina. Stanford University. Stanford, EE.UU.

Ringuelet, R. A. 1960. Rasgos fundamentales de la zoogeografía de la Argentina. Physis 22:151-170.

SaAvedra, B., y J. Simonettı. 1998. Small mammal taphonomy: intraspecific bone assemblage comparison between South and North American barn owl, Tyto alba, populations. Journal of Archaeological Science 25:165-170.

SaAvedra, B. y J. Simonettı. 2003. Holocene distribution of Octodontid rodents in central Chile. Revista Chilena de Historia Natural 76:383-389.

SaAvedra, B., D. Quiroz, y J. Iriarte. 2003. Past and present small mammals of Isla Mocha (south central Chile). Mammalian Biology 68:1-7.

Scheifler, N. A., P. Teta, y U. F. J. Pardiñas. 2012. Small mammals Didelphimorphia and Rodentia) of the archaeological site Calera (Pampean region, Buenos Aires province, Argentina): taphonomic history and Late Holocene environments. Quaternary International 278:32-44.

Short, J., y A. P. Sмith. 1994. Mammal decline and recovery in Australia. Journal of Mammalogy 75:288-297.

Silveira, M., P. Teta, V. Aldazabal, y E. Eugenio. 2010. La fauna menor en la subsistencia de los cazadores recolectores del sitio "El Divisadero Monte 6" (partido de General Lavalle, provincia de Buenos Aires). Pp. 575-581 en Zooarqueología a principios del siglo XXI. Aportes teóricos, metodológicos y casos de estudio (Gutiérrez, M., M. De Nigris, P. Fernández, M. Giardina, A. Gil, A. Izeta, G. Neme, y H. Yacobaccio, eds.). Ediciones del Espinillo. Argentina.

Simonetti, J. A. 1994. Impoverishment and nestedness in caviomorph assemblages. Journal of Mammalogy 75: 979-984.

Soriano, A., R. J. C. León, O. E. Sala, R. S. Lavado, V. A. Deregibus, M. A. Cahuepé, O. A. Scaglia, C. A. Velazquez, y J. H. Lemcoff. 1992. Río de la Plata grasslands. Pp. 367-407 en Ecosystems of the world. Natural grasslands (Coupland, R. T., ed). Elsevier. New York, EE.UU.

Stahl, P. W. 1996. The recovery and interpretation of microvertebrate bone assemblages from archaeological contexts. Journal of Archaeological Method and Theory 3:3175.

Steadman, D. W., y P. S. Martin. 2003. The late Quaternary extinction and future resurrection of birds on Pacific islands. Earth-Science Reviews 61:133-147.

Stine, S. y M. Stine. 1990.A record from Lake Cardiel of climate change in southern South America. Nature 345:705-708.

TaYlor, I. 1994. Barn Owls. Predator-prey relationships and conservation. Cambridge University Press. Cambridge, Reino Unido.

Terry, R. C . 2010. The dead don't lie: using skeletal remains for rapid assessment of historical small-mammal community baselines. Proceedings of the Royal Society B 277:1193-1201.

Teta, P., D. Loponte, y A. Acosta.2004. Sigmodontinos (Mammalia, Rodentia) del Holoceno tardío del nordeste de la provincia de Buenos Aires (Argentina). Mastozoologia Neotropical 11:69-80. 
Teta, P., A. Andrade, y U. F. J. Pardiñas. 2005. Micromamíferos (Didelphimorphia y Rodentia) y paleoambientes del Holoceno tardío en la Patagonia noroccidental extra-andina (Argentina). Archaeofauna 14:183-197.

Teta, P, C. M. González-Fischer, M. Codesido, y D. N. Bilenca. 2010. A contribution from Barn Owl pellets analysis to known micromammalian distributions in Buenos Aires province, Argentina. Mammalia 74:97-103.

Teta, P., U. F. J. Pardiñas, M. Silveira, V. Aldazabal y E. Eugenio.2013. Roedores sigmodontinos del sitio arqueológico "El Divisadero Monte6"(Holoceno tardío, Buenos Aires, Argentina): taxonomía y reconstrucción ambiental. Mastozoología Neotropical 20: 171-177.

Teta, P., U. F. J. Pardiñas, D. E. Udrizar Sauthier, y M. H. Gallardo. 2014. A new species of the tetraploid vizcacha rat Tympanoctomys (Caviomorpha, Octodontidae) from Central Patagonia, Argentina. Journal of Mammalogy 95:60-71.

Turvey, S. T. (ed.). 2009. Holocene Extinctions. Oxford University Press. Oxford, Reino Unido.

Turvey, S. T., y S. A. Fritz. 2011. The ghosts of mammals past: biological and geographical patterns of global mammalian extinction across the Holocene. Philosophical Transactions of the Royal Society of London 366:2564-2576.

Udrizar Sauthier, D. E. 2009. Los micromamíferos y la evolución ambiental durante el Holoceno en el río Chubut (Chubut, Argentina). Tesis Doctoral. Facultad de Ciencias Naturales y Museo. Universidad Nacional de La Plata. Buenos Aires, Argentina.

Udrizar Sauthier, D. E., U. F. J. Pardiñas, y E. P. Tonni. 2009. Tympanoctomys (Mammalia: Rodentia) en el Holoceno de Patagonia, Argentina. Ameghiniana 46:203-207.

Vimeaux, F., F. Sylvestre, y M. Khodri (eds.). 2009. Past Climate Variability in South America and Surrounding Regions. Springer. CIUDAD, PAÍS

Voss, R. S. 1991. On the identity of "Zygodontomys" punctulatus (Rodentia: Muroidea). American Museum Novitates 3026:1-8.

Voss, R.S., y P. Mrers. 1991. Pseudoryzomys simplex (Rodentia: Muridae) and the significance of Lund's collections from the caves of Lagoa Santa, Brazil. Bulletin American Museum of Natural History 206:414-432.

Voss, R. S., D. P. Lunde, y S. A. Jansa. 2005. On the contents of Gracilinanus Gardner and Creighton, 1989, with the description of a previously unrecognized clade of small didelphid marsupials. American Museum Novitates 3482:1-34.

Wiluams, G. 1975. The desert and the dream: a study of welsh colonization in Chubut 1865-1915. University of Wales Press. Cardiff, Gales.

Sometido: 6 de enero de 2014

Revisado: 31 de marzo de 2014

Aceptado: 12 de abril de 2014

Editor asociado: Robert Owen

Diseño gráfico editorial: Gerardo Hernández 


\section{Apéndice 1}

Abreviaturas utilizadas en las Figs. 1-4:

$\mathrm{Aa}=$ Akodon azarae; $\mathrm{Ai}=$ Akodon iniscatus; $\mathrm{Al}=$ Abrothrix longipilis; $\mathrm{Ala}=$ Abrothrix lanosa; $\mathrm{An}=$ Akodon neocenus; $\mathrm{Ao}=$ Abrothrix olivacea; $\mathrm{Bt}=$ Bibimys torresi; $\mathrm{Ca}=$ Calomys spp.; $\mathrm{Cm}=$ Chelemys macronyx; $\mathrm{Ct}=$ Ctenomys spp.; $\mathrm{El}=$ Eligmodontia spp.; Eu = Euneomys spp.; Gg = Graomys griseoflavus; GI = Galea leucoblephara; Gv = Geoxus valdivianus; $\mathrm{Hb}=$ Holochilus brasiliensis; It = Irenomys tarsalis; $\mathrm{Lh}=$ Lestodelphys halli; $\mathrm{Lm}=$ Loxodontomys micropus; $\mathrm{Ma}=$ Microcavia australis; $\mathrm{Md}=$ Monodelphis dimidiata; $\mathrm{Ne}=$ Notiomys edwardsii; $\mathrm{NI}=$ Necromys lasiurus; $\mathrm{No}=$ Necromys obscurus; $\mathrm{Ob}=$ Octodon bridgesii; Of = Oligoryzomys flavescens; Ol = Oligoryzomys longicaudatus; Or = Oxymycterus rufus; $\mathrm{Ph}=$ Phyllotis sp.; $\mathrm{Ps}=$ Pseudoryzomys simplex; $\mathrm{Px}=$ Phyllotis xanthopygus; $\mathrm{Ra}=$ Reithrodon auritus; $\mathrm{Sa}=$ Scapteromys aquaticus; $\mathrm{Tb}=$ Tadarida brasiliensis; Tk = Tympanoctomys kirchnerorum; $\mathrm{Tp}=$ Thylamys pallidior

\begin{tabular}{|c|c|c|c|c|}
\hline & $\begin{array}{l}\text { Categorización } \\
\text { UICN }\end{array}$ & $\begin{array}{l}\text { Retracción/ } \\
\text { declinación }\end{array}$ & $\begin{array}{l}\text { Expansión/aumento } \\
\text { de frecuencia }\end{array}$ & Referencia \\
\hline Abrothix longipilis & LC & si & - & Pardiñas et al. 2012 \\
\hline Abrothrix andina & LC & - & - & - \\
\hline Abrothrix lanosa & LC & si & - & Pardiñas et al. 2011 \\
\hline Abrothrix olivacea & LC & si & si & Pardiñas 1998 \\
\hline Aconaemys fuscus & LC & si & - & Saavedra y Simonetti 2003 \\
\hline Aconaemys porteri & DD & - & - & - \\
\hline Aconaemys sagei & DD & - & - & - \\
\hline Akodon albiventer & LC & - & - & - \\
\hline Akodon azarae & LC & - & - & - \\
\hline Akodon caenosus & LC & - & - & - \\
\hline Akodon neocenus & LC & - & - & - \\
\hline Akodon iniscatus & LC & si & - & Pardiñas et al. 2000, 2012 \\
\hline Akodon simulator & LC & - & - & - \\
\hline Akodon spegazzini & LC & si & - & Ortiz et al. 2011 \\
\hline Andinomys edax & LC & si & - & Ortiz et al. 2011 \\
\hline Aulyscomys sublimis & LC & - & - & - \\
\hline Bibimys torresi & NT & si & - & $\begin{array}{l}\text { Pardiñas 1999a; Teta et al. } \\
2013\end{array}$ \\
\hline Calomys boliviae & LC & - & - & - \\
\hline Calomys lepidus & LC & - & - & - \\
\hline Calomys musculinus & LC & - & si & $\begin{array}{l}\text { Pardiñas 1999a; Pardiñas et } \\
\text { al. } 2000\end{array}$ \\
\hline Calomys spp. & - & - & si & Pardiñas 1999a \\
\hline Cavia aperea & LC & - & - & - \\
\hline
\end{tabular}

Tabla 1.Taxones con registro fósil para el Holoceno más tardío en el sur de América del Sur; en negrita se indican aquellos que tienen una categoría de Preocupación menor de acuerdo a la UICN, pero que han sufrido retracciones recientes $\mathrm{y} / \mathrm{o}$ declinaciones severas en sus poblaciones en los últimos 500 años. En cada caso se indica al menos una referencia relevante. Abreviaturas para las categorías de conservación: $\mathrm{CR}=$ en peligro crítico; $\mathrm{EN}$ = en peligro; $\mathrm{EX}=$ extinto; $\mathrm{DD}=$ datos deficientes; $\mathrm{LC}=$ preocupación menor $\mathrm{NE}=$ no evaluado; $\mathrm{NT}=$ cercano a la amenaza; $\mathrm{VU}=$ vulnerable. 


\begin{tabular}{|c|c|c|c|c|}
\hline Cavia tschudii & LC & si & - & Ortiz et al. 2011 \\
\hline Chelemys macronyx & LC & si & - & Pardiñas et al. 2011 \\
\hline Ctenomys magellanicus & VU & si & - & Pardiñas et al. 2011 \\
\hline Ctenomys sociabilis & EN & si & - & Pardiñas y Teta 2013 \\
\hline Ctenomys spp. & - & si & - & $\begin{array}{l}\text { Pardiñas et al. 2012, } \\
\text { Pardiñas y Teta } 2013\end{array}$ \\
\hline Ctenomys talarum & LC & si & - & Teta et al. 2005 \\
\hline Eligmodontia cf. puerulus & $\mathrm{LC}$ & - & si & Ortiz et al. 2011 \\
\hline Eligmodontia spp. & - & - & si & Teta et al. 2005 \\
\hline Euneomys chinchilloides & LC & si & - & Pardiñas y Teta 2013 \\
\hline Euneomys mordax & LC & si & - & Tammone en prep. \\
\hline Galea leucoblephara & LC & - & - & - \\
\hline Galea tixiensis & EX & - & - & Quintana 2001b \\
\hline Geoxus valdivianus & LC & si & - & Pardiñas et al. 2005 \\
\hline Graomys griseoflavus & LC & si & - & Pardiñas et al. 2012 \\
\hline Gyldenstolpia fronto chacoensis & EN & si & - & Pardiñas et al. 2008 \\
\hline Holochilus brasiliensis & LC & si & - & Pardiñas y Teta 2011 \\
\hline Holochilus chacarius & LC & si & - & Pardiñas y Teta 2011 \\
\hline Irenomys tarsalis & $\mathrm{LC}$ & - & - & - \\
\hline Lestodelphys halli & LC & si & - & Formoso en prep. \\
\hline Loxodontomys micropus & LC & si & - & Pardiñas et al. 2012 \\
\hline Microcavia australis & $\mathrm{LC}$ & - & - & - \\
\hline Monodelphis dimidiata & LC & si & - & Teta et al. 2013 \\
\hline Necromys lasiurus & LC & si & - & Teta et al. 2013 \\
\hline Necromys obscurus & NT & si & - & Teta et al. 2013 \\
\hline Neotomys ebriosus & LC & si & - & Pardiñas et al. 2012 \\
\hline Notiomys edwardsii & LC & si & - & Pardiñas et al. 2012 \\
\hline Octodon bridgesii & VU & si & - & Saavedra y Simonetti 2003 \\
\hline Octodon degus & LC & si & - & Saavedra y Simonetti 2003 \\
\hline Octodon lunatus & NT & si & - & Saavedra y Simonetti 2003 \\
\hline Octodon pacificus & $\mathrm{CR}$ & si & - & Saavedra et al. 2003 \\
\hline Oligoryzomys brendae & DD & - & - & - \\
\hline Oligoryzomys flavescens & $\mathrm{LC}$ & - & si & Pardiñas et al. 2010a \\
\hline Oligoryzomys longicaudatus & $\mathrm{LC}$ & - & si & Pardiñas y Teta 2013 \\
\hline Oxymycterus rufus & LC & - & - & - \\
\hline Phyllotis sp. & $\mathrm{NE}$ & si & - & Pardiñas 1999a \\
\hline Phyllotis xanthopygus & $\mathrm{LC}$ & - & - & - \\
\hline Reithrodon auritus & LC & si & - & $\begin{array}{l}\text { Pardiñas 1999a, Pardiñas y } \\
\text { Teta } 2013\end{array}$ \\
\hline
\end{tabular}


MICROMAMÍFEROS E IMPACTO ANTRÓPICO

Continúa...

\begin{tabular}{|c|c|c|c|c|}
\hline Scapteromys aquaticus & LC & - & - & - \\
\hline Spalacopus cyanus & LC & si & - & Saavedra y Simonetti 2003 \\
\hline Thylamys pallidior & LC & - & si & Pardiñas et al. 2012 \\
\hline Tympanoctomys kirchnerorum & $\mathrm{NE}$ & si & - & Teta et al.2014 \\
\hline
\end{tabular}

